\title{
ON DIFFERENTIAL EQUATIONS FOR SOBOLEV-TYPE LAGUERRE POLYNOMIALS
}

\author{
J. KOEKOEK, R. KOEKOEK, AND H. BAVINCK
}

AbStraCt. The Sobolev-type Laguerre polynomials $\left\{L_{n}^{\alpha, M, N}(x)\right\}_{n=0}^{\infty}$ are orthogonal with respect to the inner product

$$
\langle f, g\rangle=\frac{1}{\Gamma(\alpha+1)} \int_{0}^{\infty} x^{\alpha} e^{-x} f(x) g(x) d x+M f(0) g(0)+N f^{\prime}(0) g^{\prime}(0),
$$

where $\alpha>-1, M \geq 0$ and $N \geq 0$.

In 1990 the first and second author showed that in the case $M>0$ and $N=0$ the polynomials are eigenfunctions of a unique differential operator of the form

$$
M \sum_{i=1}^{\infty} a_{i}(x) D^{i}+x D^{2}+(\alpha+1-x) D,
$$

where $\left\{a_{i}(x)\right\}_{i=1}^{\infty}$ are independent of $n$. This differential operator is of order $2 \alpha+4$ if $\alpha$ is a nonnegative integer, and of infinite order otherwise.

In this paper we construct all differential equations of the form

$$
\begin{aligned}
& M \sum_{i=0}^{\infty} a_{i}(x) y^{(i)}(x)+N \sum_{i=0}^{\infty} b_{i}(x) y^{(i)}(x) \\
& \quad+M N \sum_{i=0}^{\infty} c_{i}(x) y^{(i)}(x)+x y^{\prime \prime}(x)+(\alpha+1-x) y^{\prime}(x)+n y(x)=0
\end{aligned}
$$

where the coefficients $\left\{a_{i}(x)\right\}_{i=1}^{\infty},\left\{b_{i}(x)\right\}_{i=1}^{\infty}$ and $\left\{c_{i}(x)\right\}_{i=1}^{\infty}$ are independent of $n$ and the coefficients $a_{0}(x), b_{0}(x)$ and $c_{0}(x)$ are independent of $x$, satisfied by the Sobolev-type Laguerre polynomials $\left\{L_{n}^{\alpha, M, N}(x)\right\}_{n=0}^{\infty}$.

Further, we show that in the case $M=0$ and $N>0$ the polynomials are eigenfunctions of a linear differential operator, which is of order $2 \alpha+8$ if $\alpha$ is a nonnegative integer and of infinite order otherwise.

Finally, we show that in the case $M>0$ and $N>0$ the polynomials are eigenfunctions of a linear differential operator, which is of order $4 \alpha+10$ if $\alpha$ is a nonnegative integer and of infinite order otherwise.

\section{INTRODUCTION}

Let $\mathcal{P}$ denote the space of all real polynomials. We consider the polynomials $\left\{L_{n}^{\alpha, M, N}(x)\right\}_{n=0}^{\infty}$ which are orthogonal with respect to the Sobolev-type inner product

$$
\langle f, g\rangle=\frac{1}{\Gamma(\alpha+1)} \int_{0}^{\infty} x^{\alpha} e^{-x} f(x) g(x) d x+M f(0) g(0)+N f^{\prime}(0) g^{\prime}(0), f, g \in \mathcal{P},
$$

Received by the editors August 28, 1995 and, in revised form, June 24, 1996. 1991 Mathematics Subject Classification. Primary 33C45; Secondary 34A35.

Key words and phrases. Differential equations, Sobolev-type Laguerre polynomials. 
where $\alpha>-1, M \geq 0$ and $N \geq 0$. These Sobolev-type Laguerre polynomials (see [9] and [12]) are generalizations of the generalized Laguerre polynomials $\left\{L_{n}^{\alpha, M}(x)\right\}_{n=0}^{\infty}$ found by T.H. Koornwinder in [13]. They can be written as

$$
L_{n}^{\alpha, M, N}(x)=A_{0} L_{n}^{(\alpha)}(x)+A_{1} D L_{n}^{(\alpha)}(x)+A_{2} D^{2} L_{n}^{(\alpha)}(x), n=0,1,2, \ldots,
$$

where $D=\frac{d}{d x}$ denotes the differentiation operator and the coefficients $A_{0}, A_{1}$ and $A_{2}$ are given by

(2)

$$
\left\{\begin{array}{c}
A_{0}=1+M\left(\begin{array}{c}
n+\alpha \\
n-1
\end{array}\right)+\frac{n(\alpha+2)-(\alpha+1)}{(\alpha+1)(\alpha+3)} N\left(\begin{array}{c}
n+\alpha \\
n-2
\end{array}\right) \\
+\frac{M N}{(\alpha+1)(\alpha+2)}\left(\begin{array}{c}
n+\alpha \\
n-1
\end{array}\right)\left(\begin{array}{c}
n+\alpha+1 \\
n-2
\end{array}\right) \\
A_{1}=M\left(\begin{array}{c}
n+\alpha \\
n
\end{array}\right)+\frac{n-1}{\alpha+1} N\left(\begin{array}{c}
n+\alpha \\
n-1
\end{array}\right)+\frac{2 M N}{(\alpha+1)^{2}}\left(\begin{array}{c}
n+\alpha \\
n
\end{array}\right)\left(\begin{array}{c}
n+\alpha+1 \\
n-2
\end{array}\right), \\
A_{2}=\frac{N}{\alpha+1}\left(\begin{array}{c}
n+\alpha \\
n-1
\end{array}\right)+\frac{M N}{(\alpha+1)^{2}}\left(\begin{array}{c}
n+\alpha \\
n
\end{array}\right)\left(\begin{array}{c}
n+\alpha+1 \\
n-1
\end{array}\right) .
\end{array}\right.
$$

For details concerning these Sobolev-type Laguerre polynomials and their definition the reader is referred to [9] and [12].

We consider differential equations of the form

$$
\sum_{i=1}^{\infty} d_{i}(x) y^{(i)}(x)=\lambda_{n} y(x)
$$

satisfied by these Sobolev-type Laguerre polynomials, where the coefficients $\left\{d_{i}(x)\right\}_{i=1}^{\infty}$ are independent of $n$. For convenience we write these differential equations in the form

$$
\sum_{i=0}^{\infty} e_{i}(x) y^{(i)}(x)+x y^{\prime \prime}(x)+(\alpha+1-x) y^{\prime}(x)+n y(x)=0,
$$

where the coefficients $\left\{e_{i}(x)\right\}_{i=1}^{\infty}$ are independent of $n$ and the coefficient $e_{0}(x):=$ $e_{0}(n, \alpha)$ is independent of $x$.

The following lemma is well-known and easy to check. See for instance [17].

Lemma 1. Let $\left\{p_{n}(x)\right\}_{n=0}^{\infty}$ be an arbitrary set of polynomials with degree $\left[p_{n}(x)\right]=$ $n$ for each $n=0,1,2, \ldots$, and let $\left\{\lambda_{n}\right\}_{n=0}^{\infty}$ be an arbitrary sequence of constants with $\lambda_{0}=0$ and $\left\{\lambda_{n}\right\}_{n=1}^{\infty}$ not all equal to zero. Then there exists a unique sequence $\left\{d_{i}(x)\right\}_{i=1}^{\infty}$ of polynomials with degree $\left[d_{i}(x)\right] \leq i^{1}$ for all $i=1,2,3, \ldots$, such that (3) has $\left\{p_{n}(x)\right\}_{n=0}^{\infty}$ as a polynomial set of solutions. Moreover, if $d_{i}(x)=k_{i} x^{i}+$ lower order terms for $i=1,2,3, \ldots$, then

$$
n k_{1}+n(n-1) k_{2}+\ldots+n ! k_{n}=\lambda_{n}, n=1,2,3, \ldots .
$$

In [8] J. Koekoek and R. Koekoek found a differential equation of the form (3) for the generalized Laguerre polynomials $\left\{L_{n}^{\alpha, M}(x)\right\}_{n=0}^{\infty}$. These polynomials form a special case of the above mentioned Sobolev-type Laguerre polynomials, since

\footnotetext{
${ }^{1}$ The degree of the zero polynomial is supposed to be -1 .
} 
$L_{n}^{\alpha, M, 0}(x)=L_{n}^{\alpha, M}(x)$. For the symmetric form of that differential equation the reader is referred to [5].

In [1] H. Bavinck found a new method to obtain the main result of [8]. In this paper we will use this method to construct all differential equations of the form

$$
\begin{aligned}
& M \sum_{i=0}^{\infty} a_{i}(x) y^{(i)}(x)+N \sum_{i=0}^{\infty} b_{i}(x) y^{(i)}(x) \\
& \quad+M N \sum_{i=0}^{\infty} c_{i}(x) y^{(i)}(x)+x y^{\prime \prime}(x)+(\alpha+1-x) y^{\prime}(x)+n y(x)=0,
\end{aligned}
$$

where the coefficients $\left\{a_{i}(x)\right\}_{i=1}^{\infty},\left\{b_{i}(x)\right\}_{i=1}^{\infty}$ and $\left\{c_{i}(x)\right\}_{i=1}^{\infty}$ are independent of $n$ and the coefficients $a_{0}(x), b_{0}(x)$ and $c_{0}(x)$ are independent of $x$, satisfied by the polynomials $\left\{L_{n}^{\alpha, M, N}(x)\right\}_{n=0}^{\infty}$ given by (1) and (2).

In view of Lemma 1 the coefficient $M a_{i}(x)+N b_{i}(x)+M N c_{i}(x)$ must be a polynomial, independent of $n$, of degree at most $i$ for each $i=1,2,3, \ldots$ Since $M \geq$ 0 and $N \geq 0$ are arbitrary we conclude that $\left\{a_{i}(x)\right\}_{i=1}^{\infty},\left\{b_{i}(x)\right\}_{i=1}^{\infty}$ and $\left\{c_{i}(x)\right\}_{i=1}^{\infty}$ must be polynomials, independent of $n$, with degree $\left[a_{i}(x)\right] \leq i$, degree $\left[b_{i}(x)\right] \leq i$ and degree $\left[c_{i}(x)\right] \leq i$ for every $i=1,2,3, \ldots$.

The main results of this paper given in the next section (see Theorem 3) give rise to the following corollary.

Corollary 2. If $\alpha$ is a nonnegative integer, the polynomials $\left\{L_{n}^{\alpha, M, N}(x)\right\}_{n=0}^{\infty}$ satisfy a differential equation of formal order $4 \alpha+10$ which is of the form

$$
\begin{aligned}
& M \sum_{i=0}^{2 \alpha+4} a_{i}(x) y^{(i)}(x)+N \sum_{i=0}^{2 \alpha+8} \beta_{i}(x) y^{(i)}(x) \\
& \quad+M N \sum_{i=0}^{4 \alpha+10} \gamma_{i}(x) y^{(i)}(x)+x y^{\prime \prime}(x)+(\alpha+1-x) y^{\prime}(x)+n y(x)=0,
\end{aligned}
$$

where the coefficients $\left\{a_{i}(x)\right\}_{i=1}^{2 \alpha+4},\left\{\beta_{i}(x)\right\}_{i=1}^{2 \alpha+8}$ and $\left\{\gamma_{i}(x)\right\}_{i=1}^{4 \alpha+10}$ are polynomials independent of $n$ which satisfy

$$
\sum_{i=1}^{2 \alpha+4} a_{i}(x)=\sum_{i=1}^{2 \alpha+8} \beta_{i}(x)=\sum_{i=1}^{4 \alpha+10} \gamma_{i}(x)=0 .
$$

This corollary was stated as a conjecture in [11]. By formal order we mean that for special cases $(M=0$ or $N=0)$ the true order might be lower. In fact, for $M>0$ and $N=0$ we find the order $2 \alpha+4$ as in [8] and for $M=0$ and $N>0$ we find a differential equation of order $2 \alpha+8$ when $\alpha$ is a nonnegative integer.

We emphasize that except for the examples given in [11] $(\alpha=0)$ and in [10] $(\alpha=0, \alpha=1$ and $\alpha=2$ ) this is the first paper on differential equations of the form (3) satisfied by Sobolev-type orthogonal polynomials.

For more results on Sobolev orthogonality and spectral differential equations the reader is referred to [6]. Some results concerning the symmetrizability of the differential equations obtained in this paper can be found in [7].

Finally, we refer to [4] and [2], where difference operators are found for generalized Charlier polynomials, and to [3], where generalizations of Meixner polynomials are treated. In these discrete cases the difference operators turn out to be of infinite order for all values of the parameters. 
The results obtained in [8] and in this paper generalize the fourth order differential equation for the so-called Laguerre type polynomials $(\alpha=0)$ found by H.L. Krall in [15] (see also [16]). These Laguerre type polynomials are described in more detail in [14].

\section{THE MAIN RESULTS}

We look for all differential equations of the form (4) satisfied by the Sobolevtype Laguerre polynomials $\left\{L_{n}^{\alpha, M, N}(x)\right\}_{n=0}^{\infty}$ defined by (1) and (2). We emphasize that we demand that the coefficients $\left\{a_{i}(x)\right\}_{i=1}^{\infty},\left\{b_{i}(x)\right\}_{i=1}^{\infty}$ and $\left\{c_{i}(x)\right\}_{i=1}^{\infty}$ are independent of $n$ and that $a_{0}(x), b_{0}(x)$ and $c_{0}(x)$ do not depend on $x$. Sometimes we will use the notation

$$
a_{0}(x)=a_{0}(n, \alpha), b_{0}(x)=b_{0}(n, \alpha) \text { and } c_{0}(x)=c_{0}(n, \alpha), n=0,1,2, \ldots,
$$

in order to denote the dependence of $n$.

We will prove that the coefficients $\left\{a_{i}(x)\right\}_{i=0}^{\infty}$ are given by

$$
a_{0}(x)=a_{0}(n, \alpha)=\left(\begin{array}{c}
n+\alpha+1 \\
n-1
\end{array}\right), n=0,1,2, \ldots,
$$

and

$$
a_{i}(x)=\frac{1}{i !} \sum_{j=1}^{i}(-1)^{i+j+1}\left(\begin{array}{c}
\alpha+1 \\
j-1
\end{array}\right)\left(\begin{array}{c}
\alpha+2 \\
i-j
\end{array}\right)(\alpha+3)_{i-j} x^{j}, i=1,2,3, \ldots
$$

This was already found in [8] and also in [1].

From (6) it is not very difficult to see that, for $\alpha \neq 0,1,2, \ldots$,

$$
\text { degree }\left[a_{i}(x)\right]=i, i=1,2,3, \ldots,
$$

and for nonnegative integer values of $\alpha$

$$
\begin{cases}\operatorname{degree}\left[a_{i}(x)\right]=i, & i=1,2,3, \ldots, \alpha+2, \\ \operatorname{degree}\left[a_{i}(x)\right]=\alpha+2, & i=\alpha+3, \alpha+4, \alpha+5, \ldots, 2 \alpha+4, \\ a_{i}(x)=0, & i>2 \alpha+4 .\end{cases}
$$

Note that we have

$$
a_{2 \alpha+4}(x)=(-1)^{\alpha+1} \frac{x^{\alpha+2}}{(\alpha+2) !} \text { for } \alpha \in\{0,1,2, \ldots\} .
$$

This implies that, for $M>0$ the differential equation given by

$$
M \sum_{i=0}^{\infty} a_{i}(x) y^{(i)}(x)+x y^{\prime \prime}(x)+(\alpha+1-x) y^{\prime}(x)+n y(x)=0,
$$

satisfied by the polynomials $\left\{L_{n}^{\alpha, M}(x)\right\}_{n=0}^{\infty}$, where the coefficients $\left\{a_{i}(x)\right\}_{i=0}^{\infty}$ are given by (5) and (6), has order $2 \alpha+4$ if $\alpha$ is a nonnegative integer and has infinite order otherwise. 
The coefficients $\left\{b_{i}(x)\right\}_{i=0}^{\infty}$ and $\left\{c_{i}(x)\right\}_{i=0}^{\infty}$ are not unique. In fact we will show that

$$
\left\{\begin{array}{l}
b_{0}(0, \alpha)=0, \\
b_{0}(n, \alpha)=b_{0}(1, \alpha)+\beta_{0}(n, \alpha), n=1,2,3, \ldots, \\
b_{i}(x)=b_{0}(1, \alpha) b_{i}^{*}(x)+\beta_{i}(x), i=1,2,3, \ldots,
\end{array}\right.
$$

and

$$
\left\{\begin{array}{l}
c_{0}(0, \alpha)=0, \\
c_{0}(n, \alpha)=b_{0}(1, \alpha)+\gamma_{0}(n, \alpha), n=1,2,3, \ldots, \\
c_{i}(x)=b_{0}(1, \alpha) c_{i}^{*}(x)+\gamma_{i}(x), i=1,2,3, \ldots,
\end{array}\right.
$$

where $b_{0}(1, \alpha)$ is arbitrary. Here we have (cf. [10] and [11])

$$
b_{i}^{*}(x)=\frac{1}{i !} \sum_{j=0}^{i}(-1)^{j}\left(\begin{array}{l}
i \\
j
\end{array}\right)(\alpha+1)_{i-j} x^{j}, i=1,2,3, \ldots,
$$

and

$$
c_{i}^{*}(x)=\frac{(-1)^{i}}{i !} x^{i}, i=1,2,3, \ldots
$$

Further, we will show that the coefficients $\left\{\beta_{i}(x)\right\}_{i=0}^{\infty}$ are given by

$$
\beta_{0}(x)=\beta_{0}(n, \alpha)=\frac{n(\alpha+2)-\alpha}{(\alpha+1)(\alpha+4)}\left(\begin{array}{c}
n+\alpha+1 \\
n-2
\end{array}\right), n=1,2,3, \ldots,
$$

$$
\beta_{1}(x)=0 \text { and } \beta_{i}(x)=\beta_{i}^{(1)}(x)+\beta_{i}^{(2)}(x)+\beta_{i}^{(3)}(x)+\beta_{i}^{(4)}(x), i=2,3,4, \ldots,
$$

where for $i=2,3,4, \ldots$

$$
\beta_{i}^{(1)}(x)=\frac{1}{(\alpha+1)(i-2) !} \sum_{j=1}^{i}(-1)^{i+j+1}\left(\begin{array}{c}
\alpha+2 \\
j-1
\end{array}\right)\left(\begin{array}{c}
\alpha+3 \\
i-j
\end{array}\right)(\alpha+2)_{i-j} x^{j-1}
$$

$$
\begin{aligned}
& \beta_{i}^{(2)}(x)=\frac{2(\alpha+2)}{(\alpha+1)(i-1) !} \sum_{j=2}^{i}(-1)^{i+j+1}\left(\begin{array}{c}
\alpha+1 \\
j-2
\end{array}\right)\left(\begin{array}{c}
\alpha+2 \\
i-j
\end{array}\right)(\alpha+3)_{i-j} x^{j-2} \\
& \beta_{i}^{(3)}(x)=\frac{1}{(\alpha+1)^{2}(\alpha+2)(\alpha+3)^{2}(i-2) !} \sum_{j=0}^{i}(-1)^{i+j+1}\left(\begin{array}{c}
\alpha+3 \\
j
\end{array}\right)\left(\begin{array}{c}
\alpha+3 \\
i-j
\end{array}\right)
\end{aligned}
$$

$$
\times(\alpha+1)_{i-j}[(\alpha+1)(\alpha+3)+j(i-j)] x^{j},
$$

and

$$
\beta_{i}^{(4)}(x)=\frac{i-2}{(\alpha+1)^{2}(\alpha+3)(\alpha+4)^{2}(i-2) !} \sum_{j=0}^{i}(-1)^{i+j+1}\left(\begin{array}{c}
\alpha+4 \\
j
\end{array}\right)\left(\begin{array}{c}
\alpha+4 \\
i-j
\end{array}\right)
$$

$$
\times(\alpha+1)_{i-j}[(\alpha+1)(\alpha+4)+j(i-j)] x^{j},
$$


From (12), (13), (14), (15) and (16) we obtain that for $\alpha \neq 0,1,2, \ldots$

$$
\text { degree }\left[\beta_{i}(x)\right]=i, i=2,3,4, \ldots,
$$

and for nonnegative integer values of $\alpha$

$$
\begin{cases}\operatorname{degree}\left[\beta_{i}(x)\right]=i, & i=2,3,4, \ldots, \alpha+4, \\ \operatorname{degree}\left[\beta_{i}(x)\right]=\alpha+4, & i=\alpha+5, \alpha+6, \alpha+7, \ldots, 2 \alpha+8, \\ \beta_{i}(x)=0, & i>2 \alpha+8 .\end{cases}
$$

We remark that

$$
\beta_{2 \alpha+8}(x)=\beta_{2 \alpha+8}^{(4)}(x)=(-1)^{\alpha+1} \frac{\alpha+2}{\alpha+1} \frac{x^{\alpha+4}}{(\alpha+4) !} \text { for } \alpha \in\{0,1,2, \ldots\} .
$$

This implies that for $N>0$ the differential equation

$$
N \sum_{i=0}^{\infty} \beta_{i}(x) y^{(i)}(x)+x y^{\prime \prime}(x)+(\alpha+1-x) y^{\prime}(x)+n y(x)=0,
$$

satisfied by the polynomials $\left\{L_{n}^{\alpha, 0, N}(x)\right\}_{n=0}^{\infty}$, where the coefficients $\left\{\beta_{i}(x)\right\}_{i=0}^{\infty}$ are given by $\beta_{0}(0, \alpha)=0$ and (11), (12), (13), (14), (15) and (16), has order $2 \alpha+8$ if $\alpha$ is a nonnegative integer and has infinite order otherwise.

Finally we will show that the coefficients $\left\{\gamma_{i}(x)\right\}_{i=0}^{\infty}$ are given by

$$
\gamma_{0}(x)=\gamma_{0}(n, \alpha)=\frac{1}{\alpha+1}\left(\begin{array}{c}
n+\alpha+1 \\
n-2
\end{array}\right){ }_{3} F_{2}\left(\begin{array}{c}
-n+2,-\alpha-2, \alpha+3 \mid \\
2, \alpha+4
\end{array}\right),
$$

and

$$
\gamma_{i}(x)=\gamma_{i}^{(1)}(x)+\gamma_{i}^{(2)}(x)+\gamma_{i}^{(3)}(x)+\gamma_{i}^{(4)}(x)+\gamma_{i}^{(5)}(x), i=1,2,3, \ldots,
$$

where for $i=1,2,3, \ldots$

$$
\gamma_{i}^{(1)}(x)=\frac{(-1)^{i+1}}{(\alpha+1)(i-1) !} \sum_{k=1}^{i} a_{k}(x) \sum_{j=k}^{i}(-1)^{j}\left(\begin{array}{c}
\alpha+3 \\
j-k
\end{array}\right)\left(\begin{array}{c}
\alpha+3 \\
i-j
\end{array}\right)(\alpha+4)_{i-j+k-1} x^{j-k}
$$

$$
\begin{gathered}
\gamma_{i}^{(2)}(x)=\frac{(-1)^{i}}{(i+1) !} \sum_{k=1}^{i} \beta_{k}(x) \sum_{j=k}^{i}(-1)^{j}\left(\begin{array}{c}
\alpha+1 \\
j-k
\end{array}\right)\left(\begin{array}{c}
\alpha+1 \\
i-j
\end{array}\right)(\alpha+3)_{i-j+k} x^{j-k}, \\
\gamma_{i}^{(3)}(x)=\frac{2}{(\alpha+1)(\alpha+3)} \sum_{j=0}^{i} \sum_{n=0}^{j}(-1)^{j} \frac{(-j)_{n}(\alpha+3)_{i-j+n}(\alpha+3)_{n}}{j !(i-j) ! n !}
\end{gathered}
$$

$$
\times \sum_{k=0}^{i-j} \frac{(-i+j)_{k}(n+\alpha+3)_{k}(n+\alpha+4)_{k}}{\Gamma(n+k) \Gamma(n+k+2) k !} x^{j}
$$




$$
\gamma_{i}^{(4)}(x)=-\frac{2}{(\alpha+1)(\alpha+4)} \sum_{j=0}^{i} \sum_{n=0}^{j}(-1)^{j} \frac{(-j)_{n}(\alpha+3)_{i-j+n}(\alpha+4)_{n}}{j !(i-j) ! n !}
$$

$$
\times \sum_{k=0}^{i-j} \frac{(-i+j)_{k}(n+\alpha+3)_{k}(n+\alpha+4)_{k}}{\Gamma(n+k) \Gamma(n+k+2) k !} x^{j}
$$

and

$$
\begin{aligned}
& \gamma_{i}^{(5)}(x)=\frac{(-1)^{i+1}}{(\alpha+1)(\alpha+3)} \sum_{k=0}^{i-1}(-1)^{k} \frac{(-\alpha-2)_{k}(\alpha+3)_{k}}{(2)_{k}(\alpha+4)_{k} k !} \\
& \times \frac{1}{\Gamma(i-k)} \sum_{j=0}^{i}(-1)^{j}\left(\begin{array}{c}
k+\alpha+3 \\
j
\end{array}\right)\left(\begin{array}{c}
k+\alpha+3 \\
i-j
\end{array}\right)(\alpha+3)_{i-j} x^{j} .
\end{aligned}
$$

From (18), (19), (20), (21), (22) and (23) we obtain that $\gamma_{1}(x)=0$.

Moreover we will show that, for nonnegative integer values of $\alpha$,

$$
\gamma_{i}(x)=0 \text { for } i>4 \alpha+10
$$

and that

$$
\gamma_{4 \alpha+10}(x)=\gamma_{4 \alpha+10}^{(5)}(x)=\frac{x^{2 \alpha+5}}{(\alpha+1)(2 \alpha+5)(\alpha+2) !(\alpha+3) !} \text { for } \alpha \in\{0,1,2, \ldots\} .
$$

Note that for $i \geq 3$ the coefficient of $y^{(i)}(x)$ in the differential equation (4) is equal to $M a_{i}(x)+N b_{i}(x)+M N c_{i}(x)$. Hence for $M^{2}+N^{2}>0$ finite order can only occur when $M a_{i}(x), N b_{i}(x)$ and $M N c_{i}(x)$ all vanish for $i \geq K$ for some $K \in\{3,4,5, \ldots\}$.

Now we have reached our main theorem:

Theorem 3. For $\alpha>-1$ and $M^{2}+N^{2}>0$ the only differential equations of the form (4) satisfied by the Sobolev-type Laguerre polynomials $\left\{L_{n}^{\alpha, M, N}(x)\right\}_{n=0}^{\infty}$ defined by (1) and (2), are those where the coefficients $\left\{a_{i}(x)\right\}_{i=0}^{\infty},\left\{b_{i}(x)\right\}_{i=0}^{\infty}$ and $\left\{c_{i}(x)\right\}_{i=0}^{\infty}$ are given by (5) up to and including (23) with $b_{0}(1, \alpha)$ arbitrary.

Only if $N b_{0}(1, \alpha)=0$ and $\alpha \in\{0,1,2, \ldots\}$ is the order of this differential equation finite, and equal to

$$
\begin{cases}2 \alpha+4 & \text { if } \quad M>0 \text { and } N=0 \\ 2 \alpha+8 & \text { if } \quad M=0 \text { and } N>0 \\ 4 \alpha+10 & \text { if } \quad M>0 \text { and } N>0\end{cases}
$$

Otherwise the differential equation is of infinite order.

Finally we will show that the coefficients $\left\{a_{i}(x)\right\}_{i=1}^{\infty},\left\{\beta_{i}(x)\right\}_{i=1}^{\infty}$ and $\left\{\gamma_{i}(x)\right\}_{i=1}^{\infty}$ satisfy

$$
\begin{aligned}
& \sum_{i=1}^{\infty} a_{i}(x)=-\frac{\sin \pi \alpha}{\pi} \frac{x}{(\alpha+2)(\alpha+3)}{ }_{1} F_{1}\left(\begin{array}{c}
1 \\
\alpha+4
\end{array} \mid-x\right) \\
& \sum_{i=1}^{\infty} \beta_{i}(x)=\frac{\sin \pi \alpha}{\pi} \frac{\alpha+2}{(\alpha+1)(\alpha+3)(\alpha+4)}\left[1-x_{2} F_{2}\left(\begin{array}{c}
1, \alpha+3 \\
\alpha+2, \alpha+5
\end{array} \mid-x\right)\right],
\end{aligned}
$$


$\alpha>-1$, and

$$
\sum_{i=1}^{\infty} \gamma_{i}(x)=0 \text { for } \alpha \in\{0,1,2, \ldots\}
$$

This implies that for nonnegative integer values of $\alpha$ we have

$$
\sum_{i=1}^{2 \alpha+4} a_{i}(x)=\sum_{i=1}^{2 \alpha+8} \beta_{i}(x)=\sum_{i=1}^{4 \alpha+10} \gamma_{i}(x)=0
$$

\section{Some Classical FORMUlas}

We start with the following lemma on partial sums of Gauss' hypergeometric series which will be used in this paper:

Lemma 4. Let $n \in\{0,1,2, \ldots\}$. Then the nth partial sum of the Gauss' hypergeometric series at the point 1 ,

$$
\sum_{k=0}^{\infty} \frac{(a)_{k}(b)_{k}}{(c)_{k} k !}
$$

equals

$$
\sum_{k=0}^{n} \frac{(a)_{k}(b)_{k}}{(c)_{k} k !}=\frac{(a+1)_{n}}{n !}{ }_{3} F_{2}\left(\begin{array}{c}
-n, a, c-b \\
a+1, c
\end{array} \mid 1\right)
$$

for all values of the parameters $a, b$ and $c$ for which $(a+1)_{n}(c)_{n} \neq 0$.

Proof. The proof is based on the well-known Vandermonde summation formula

$$
{ }_{2} F_{1}\left(\begin{array}{c|c}
-n, b \\
c
\end{array} \mid 1\right)=\frac{(c-b)_{n}}{(c)_{n}},(c)_{n} \neq 0, n=0,1,2, \ldots .
$$

Suppose that $n \in\{0,1,2, \ldots\}$. Then for $(a+1)_{n}(c)_{n} \neq 0$, by changing the order of summation and applying (24) twice, we find that

$$
\begin{aligned}
& { }_{3} F_{2}\left(\begin{array}{c}
-n, a, c-b \\
a+1, c
\end{array} \mid 1\right)=\sum_{m=0}^{n} \frac{(-n)_{m}(a)_{m}}{(a+1)_{m} m !} F_{1}\left(\begin{array}{c}
-m, b \\
c
\end{array} \mid 1\right) \\
= & \sum_{m=0}^{n} \sum_{k=0}^{m} \frac{(-n)_{m}(a)_{m}}{(a+1)_{m} m !} \frac{(-m)_{k}(b)_{k}}{(c)_{k} k !}=\sum_{k=0}^{n} \sum_{m=k}^{n} \frac{(-n)_{m}(a)_{m}(-m)_{k}(b)_{k}}{(a+1)_{m}(c)_{k} m ! k !} \\
= & \sum_{k=0}^{n} \sum_{m=0}^{n-k} \frac{(-n)_{k+m}(a)_{k+m}(-k-m)_{k}(b)_{k}}{(a+1)_{k+m}(c)_{k}(k+m) ! k !} \\
= & \sum_{k=0}^{n} \frac{(-n)_{k}(a)_{k}(b)_{k}}{(a+1)_{k}(c)_{k} k !}(-1)^{k} F_{1}\left(\begin{array}{c}
-n+k, a+k \\
a+k+1
\end{array} \mid 1\right) \\
= & \sum_{k=0}^{n} \frac{(-n)_{k}(a)_{k}(b)_{k}}{(a+1)_{k}(c)_{k} k !}(-1)^{k} \frac{(1)_{n-k}}{(a+k+1)_{n-k}}=\frac{n !}{(\alpha+1)_{n}} \sum_{k=0}^{n} \frac{(a)_{k}(b)_{k}}{(c)_{k} k !} .
\end{aligned}
$$

This proves Lemma 4.

The special case $b=c$ of Lemma 4 leads to the $n$th partial sum of a ${ }_{1} F_{0}$ hypergeometric series at the point 1 :

$$
\sum_{k=0}^{n}\left(\begin{array}{c}
a+k \\
k
\end{array}\right)=\sum_{k=0}^{n} \frac{(a+1)_{k}}{k !}=\frac{(a+2)_{n}}{n !}=\left(\begin{array}{c}
n+a+1 \\
n
\end{array}\right), n=0,1,2, \ldots
$$


We list some definitions and properties of the classical Laguerre polynomials $\left\{L_{n}^{(\alpha)}(x)\right\}_{n=0}^{\infty}$ which we need in this paper.

For $\alpha$ real and $\alpha>-1$ the classical Laguerre polynomials are orthogonal on the interval $[0, \infty)$ with respect to the weight function $x^{\alpha} e^{-x}$. They are usually defined by

$$
L_{n}^{(\alpha)}(x)=\left(\begin{array}{c}
n+\alpha \\
n
\end{array}\right){ }_{1} F_{1}\left(\begin{array}{c}
-n \\
\alpha+1
\end{array} \mid x\right), n=0,1,2, \ldots
$$

Note that $L_{n}^{(\alpha)}(x)$ is also a polynomial in the parameter $\alpha$. This implies that the classical Laguerre polynomials can be defined for all $\alpha$ by

$$
\begin{aligned}
L_{n}^{(\alpha)}(x) & =\frac{1}{n !} \sum_{k=0}^{n}(-n)_{k}(\alpha+k+1)_{n-k} \frac{x^{k}}{k !} \\
& =(-1)^{n} \sum_{k=0}^{n} \frac{(-n-\alpha)_{n-k}}{(n-k) !} \frac{x^{k}}{k !} \\
& =\sum_{k=0}^{n}(-1)^{k} \frac{(\alpha+k+1)_{n-k}}{(n-k) !} \frac{x^{k}}{k !}, n=0,1,2, \ldots .
\end{aligned}
$$

Now we take $\alpha$ arbitrary. We have

$$
L_{n}^{(\alpha)}(0)=\left(\begin{array}{c}
n+\alpha \\
n
\end{array}\right)=\frac{(\alpha+1)_{n}}{n !}, n=0,1,2, \ldots,
$$

and

$$
D^{k} L_{n}^{(\alpha)}(x)=(-1)^{k} L_{n-k}^{(\alpha+k)}(x), k \leq n, k, n=0,1,2, \ldots .
$$

The classical Laguerre polynomials satisfy the second order linear differential equation

$$
x y^{\prime \prime}(x)+(\alpha+1-x) y^{\prime}(x)+n y(x)=0 .
$$

From the generating function given by

$$
\sum_{n=0}^{\infty} L_{n}^{(\alpha)}(x) t^{n}=(1-t)^{-\alpha-1} \exp \left(\frac{x t}{t-1}\right)
$$

it easily follows that for arbitrary $p$ we have

$$
L_{n}^{(\alpha-p)}(x)=\sum_{k=0}^{n}(-1)^{k}\left(\begin{array}{l}
p \\
k
\end{array}\right) L_{n-k}^{(\alpha)}(x), n=0,1,2, \ldots .
$$

Further we have for $i=0,1,2, \ldots$

$$
D^{i} L_{n}^{(\alpha)}(x)=D^{i+1} L_{n}^{(\alpha)}(x)-D^{i+1} L_{n+1}^{(\alpha)}(x), n=0,1,2, \ldots,
$$

or equivalently

$$
D^{i+1} L_{n+1}^{(\alpha)}(x)=D^{i+1} L_{n}^{(\alpha)}(x)-D^{i} L_{n}^{(\alpha)}(x), n=0,1,2, \ldots .
$$

We also obtain from the generating function that

$$
\sum_{k=0}^{\infty} L_{k}^{(-\alpha-i-1)}(-x) t^{k} \sum_{m=0}^{\infty} L_{m}^{(\alpha+j)}(x) t^{m}=(1-t)^{i-j-1} .
$$


This implies that

$$
\sum_{k=j}^{i} L_{i-k}^{(-\alpha-i-1)}(-x) L_{k-j}^{(\alpha+j)}(x)=\delta_{i j}, j \leq i, i, j=0,1,2, \ldots .
$$

This inversion formula is an important tool to obtain the results of this paper. We will use it in the following way:

Lemma 5. Suppose that for $k \in\{0,1,2, \ldots\}$ we have the system of equations

$$
\sum_{i=1}^{\infty} A_{i}(x) D^{i+k} L_{n}^{(\alpha)}(x)=F_{n}(x), n=k+1, k+2, k+3, \ldots,
$$

where $\left\{A_{i}(x)\right\}_{i=1}^{\infty}$ are independent of $n$. Then this system has a unique solution given by

$$
A_{i}(x)=(-1)^{i+k} \sum_{j=1}^{i} L_{i-j}^{(-\alpha-i-k-1)}(-x) F_{j+k}(x), i=1,2,3, \ldots .
$$

Finally we define the following polynomials involving the classical Laguerre polynomials:

$$
\begin{gathered}
F_{i}(a, b ; c ; x):=\sum_{k=0}^{i} \frac{(a)_{k}}{\Gamma(c+k)} L_{i-k}^{(-a-i)}(-x) L_{k}^{(b)}(x), i=0,1,2, \ldots, \\
G_{i}(a, b ; c ; x):=\sum_{k=0}^{i} \frac{(a)_{k}}{\Gamma(c+k)} L_{i-k}^{(-b-i-1)}(-x) L_{k}^{(b)}(x), i=0,1,2, \ldots,
\end{gathered}
$$

and

$$
H_{i}(a, b, c ; d, e ; x):=\sum_{k=0}^{i} \frac{(a)_{k}(b)_{k}}{\Gamma(d+k) \Gamma(e+k)} L_{i-k}^{(-a-i)}(-x) L_{k}^{(c)}(x), i=0,1,2, \ldots
$$

Note that $F_{i}$ and $G_{i}$ are polynomials in $a, b$ and $x$ and that $H_{i}$ is a polynomial in $a, b, c$ and $x$ for each $i=0,1,2, \ldots$.

Now we will prove the following lemma:

\section{Lemma 6.}

(40)

$$
F_{i}(a, b ; c ; x)=\frac{1}{\Gamma(c+i)} \sum_{j=0}^{i}(-1)^{j}\left(\begin{array}{c}
a-c \\
j
\end{array}\right)\left(\begin{array}{c}
b-c+1 \\
i-j
\end{array}\right)(a)_{i-j} x^{j}, i=0,1,2, \ldots,
$$

and

$$
\begin{aligned}
& G_{i}(a+2, a ; c ; x) \\
= & \frac{1}{(a+1)(a-c+2) \Gamma(c+i)} \sum_{j=0}^{i}(-1)^{j}\left(\begin{array}{c}
a-c+2 \\
j
\end{array}\right)\left(\begin{array}{c}
a-c+2 \\
i-j
\end{array}\right)(a+1)_{i-j}
\end{aligned}
$$

$$
\times[(a+1)(a-c+2)+j(i-j)] x^{j}, i=0,1,2, \ldots
$$


Proof. First we define (see for instance [19])

$$
{ }_{2} \Phi_{1}\left(\begin{array}{c}
a, b \\
c
\end{array} \mid z\right):=\sum_{k=0}^{\infty} \frac{(a)_{k}(b)_{k}}{\Gamma(c+k) k !} z^{k}
$$

for all complex $a, b, c$ and $z$ for which the series in the right-hand side converges. If $c \neq 0,-1,-2, \ldots$ we may write

$$
{ }_{2} \Phi_{1}\left(\begin{array}{c|c}
a, b \\
c
\end{array} \mid z\right)=\frac{1}{\Gamma(c)}{ }_{2} F_{1}\left(\begin{array}{c}
a, b \\
c
\end{array} \mid z\right)
$$

Now we have (cf. (24))

$$
{ }_{2} \Phi_{1}\left(\begin{array}{c|c}
-n, b & 1 \\
c & 1
\end{array}\right)=\frac{(c-b)_{n}}{\Gamma(c+n)}, n=0,1,2, \ldots
$$

for all complex values of $b$ and $c$. This is the well-known Vandermonde summation formula.

If we apply definition (28) to $L_{i-k}^{(-a-i)}(-x)$ and definition (29) to $L_{k}^{(b)}(x)$ in (37), change the order of the summations and apply (42) twice, we obtain for each $i=$ $0,1,2, \ldots$

$$
\begin{aligned}
F_{i} & (a, b ; c ; x)=\sum_{k=0}^{i} \sum_{m=0}^{i-k} \sum_{n=0}^{k}(-1)^{i+n} \frac{(a)_{k}(-i)_{k+m}(a+k)_{i-k-m}(n+b+1)_{k-n}}{\Gamma(c+k) i ! m !(k-n) ! n !} x^{m+n} \\
& =\sum_{m=0}^{i} \sum_{n=0}^{i-m} \sum_{k=0}^{i-m-n}(-1)^{i+n} \frac{(-i)_{m+n+k}(a)_{i-m}(n+b+1)_{k}}{\Gamma(c+n+k) i ! m ! n ! k !} x^{m+n} \\
& =\sum_{m=0}^{i} \sum_{n=0}^{i-m}(-1)^{i+n} \frac{(-i)_{m+n}(a)_{i-m}}{i ! m ! n !}{ }_{2} \Phi_{1}\left(\begin{array}{c}
-i+m+n, n+b+1 \\
c+n
\end{array}\right) x^{m+n} \\
& =\sum_{j=0}^{i} \sum_{n=0}^{j}(-1)^{i+n} \frac{(-i)_{j}(a)_{i-j+n}(c-b-1)_{i-j}}{i !(j-n) ! n ! \Gamma(c+i-j+n)} x^{j} \\
& =\frac{(-1)^{i}}{i !} \sum_{j=0}^{i} \frac{(-i)_{j}(a)_{i-j}(c-b-1)_{i-j}}{j !}{ }_{2} \Phi_{1}\left(\begin{array}{c}
-j, a+i-j \\
c+i-j
\end{array} \mid 1\right) x^{j} \\
& =\frac{(-1)^{i}}{i ! \Gamma(c+i)} \sum_{j=0}^{i}(-i)_{j}(c-a)_{j}(a)_{i-j}(c-b-1)_{i-j} \frac{x^{j}}{j !} \\
& =\frac{1}{\Gamma(c+i)} \sum_{j=0}^{i}(-1)^{j}\left(\begin{array}{c}
a-c \\
j
\end{array}\right)\left(\begin{array}{c}
b-c+1 \\
i-j
\end{array}\right)(a)_{i-j} x^{j},
\end{aligned}
$$

which proves (40). 
In a similar way we obtain for each $i=0,1,2, \ldots$

$$
\begin{aligned}
G_{i} & (a+2, a ; c ; x)=\sum_{k=0}^{i} \sum_{m=0}^{i-k} \sum_{n=0}^{k}(-1)^{i+n} \frac{(-i)_{k+m}(a+2)_{k}(n+a+1)_{i-m-n}}{\Gamma(c+k) i ! m !(k-n) ! n !} x^{m+n} \\
= & \sum_{m=0}^{i} \sum_{n=0}^{i-m} \sum_{k=0}^{i-m-n}(-1)^{i+n} \frac{(-i)_{m+n+k}(a+2)_{n+k}(n+a+1)_{i-m-n}}{\Gamma(c+n+k) i ! m ! n ! k !} x^{m+n} \\
= & \sum_{m=0}^{i} \sum_{n=0}^{i-m}(-1)^{i+n} \frac{(-i)_{m+n}(a+2)_{n}(n+a+1)_{i-m-n}}{i ! m ! n !} \\
= & \sum_{j=0}^{i} \sum_{n=0}^{j}(-1)^{i+n} \frac{(-i)_{j}(a+2)_{n}(n+a+1)_{i-j}(c-a-2)_{i-j}}{i !(j-n) ! n ! \Gamma(c+i-j+n)} x^{j} \\
= & \frac{(-1)^{i}}{i !} \sum_{j=0}^{i}(-i)_{j}(c-a-2)_{i-j} \frac{x^{j}}{j !} \sum_{n=0}^{j} \frac{(-j)_{n}(a+2)_{n}(n+a+1)_{i-j}}{n ! \Gamma(c+i-j+n)} .
\end{aligned}
$$

Now we use (42) to find that, for $j=1,2,3, \ldots$,

$$
\begin{aligned}
& \sum_{n=0}^{j} \frac{(-j)_{n}(a+2)_{n}(n+a+1)_{i-j}}{n ! \Gamma(c+i-j+n)}=\sum_{n=0}^{j} \frac{(-j)_{n}(a+1+n)(a+1)_{i-j+n}}{(a+1) n ! \Gamma(c+i-j+n)} \\
& =(a+1)_{i-j} \sum_{n=0}^{j} \frac{(-j)_{n}(a+i-j+1)_{n}}{n ! \Gamma(c+i-j+n)}+(a+2)_{i-j} \sum_{n=1}^{j} \frac{(-j)_{n}(a+i-j+2)_{n-1}}{(n-1) ! \Gamma(c+i-j+n)} \\
& =(a+1)_{i-j 2} \Phi_{1}\left(\begin{array}{c}
-j, a+i-j+1 \\
c+i-j
\end{array} \mid 1\right) \\
& =\frac{(a+1)_{i-j}(c-a-2)_{j}}{(a+1)(a-c+2) \Gamma(c+i)}[(a+1)(a-c+2-j)+j(a+1+i-j)] .
\end{aligned}
$$

Note that this result is also true for $j=0$. Hence, for $i=0,1,2, \ldots$ we obtain

$$
\begin{aligned}
& G_{i}(a+2, a ; c ; x) \\
& =\frac{(-1)^{i}}{(a+1)(a-c+2) i ! \Gamma(c+i)} \sum_{j=0}^{i}(-i)_{j}(c-a-2)_{j}(c-a-2)_{i-j}(a+1)_{i-j} \\
& \times[(a+1)(a-c+2-j)+j(a+1+i-j)] \frac{x^{j}}{j !} \\
& =\frac{1}{(a+1)(a-c+2) \Gamma(c+i)} \sum_{j=0}^{i}(-1)^{j}\left(\begin{array}{c}
a-c+2 \\
j
\end{array}\right)\left(\begin{array}{c}
a-c+2 \\
i-j
\end{array}\right)(a+1)_{i-j} \\
& \times[(a+1)(a-c+2)+j(i-j)] x^{j},
\end{aligned}
$$

which proves (41). 
If we also define (cf. [19])

$$
{ }_{3} \Phi_{2}\left(\begin{array}{c}
a, b, c \\
d, e
\end{array} \mid z\right):=\sum_{k=0}^{\infty} \frac{(a)_{k}(b)_{k}(c)_{k}}{\Gamma(d+k) \Gamma(e+k) k !} z^{k},
$$

for all complex $a, b, c, d, e$ and $z$ for which the series in the right-hand side converges, then we also have

\section{Lemma 7.}

$$
\begin{aligned}
H_{i}(a, b, c ; d, e ; x)=\sum_{j=0}^{i} & \sum_{n=0}^{j}(-1)^{i+j} \frac{(-j)_{n}(a)_{i-j+n}(b)_{n}}{j !(i-j) ! n !} \\
& \times{ }_{3} \Phi_{2}\left(\begin{array}{c}
-i+j, n+b, n+c+1 \\
n+d, n+e
\end{array} \mid 1\right) x^{j}, i=0,1,2, \ldots .
\end{aligned}
$$

Proof. As before we apply definition (28) to $L_{i-k}^{(-a-i)}(-x)$ and definition (29) to $L_{k}^{(c)}(x)$ in (39) and change the order of the summations to obtain, for each $i=$ $0,1,2, \ldots$,

$$
\begin{aligned}
& H_{i}(a, b, c ; d, e ; x) \\
& =\sum_{k=0}^{i} \sum_{m=0}^{i-k} \sum_{n=0}^{k}(-1)^{i+n} \frac{(-i)_{k+m}(a)_{i-m}(b)_{k}(n+c+1)_{k-n}}{\Gamma(d+k) \Gamma(e+k) i ! m !(k-n) ! n !} x^{m+n} \\
& =\sum_{m=0}^{i} \sum_{n=0}^{i-m} \sum_{k=0}^{i-m-n}(-1)^{i+n} \frac{(-i)_{m+n+k}(a)_{i-m}(b)_{n+k}(n+c+1)_{k}}{\Gamma(d+n+k) \Gamma(e+n+k) i ! m ! n ! k !} x^{m+n} \\
& =\sum_{m=0}^{i} \sum_{n=0}^{i-m}(-1)^{i+n} \frac{(-i)_{m+n}(a)_{i-m}(b)_{n}}{i ! m ! n !} \\
& \times{ }_{3} \Phi_{2}\left(\begin{array}{c|c}
-i+m+n, n+b, n+c+1 & 1 \\
n+d, n+e & 1
\end{array}\right) x^{m+n} \\
& =\sum_{j=0}^{i} \sum_{n=0}^{j}(-1)^{i+n} \frac{(-i)_{j}(a)_{i-j+n}(b)_{n}}{i !(j-n) ! n !}{ }_{3} \Phi_{2}\left(\begin{array}{c}
-i+j, n+b, n+c+1 \\
n+d, n+e
\end{array} \mid 1\right) x^{j} \\
& =\sum_{j=0}^{i} \sum_{n=0}^{j}(-1)^{i+j} \frac{(-j)_{n}(a)_{i-j+n}(b)_{n}}{j !(i-j) ! n !}{ }_{3} \Phi_{2}\left(\begin{array}{c}
-i+j, n+b, n+c+1 \\
n+d, n+e
\end{array} \mid 1\right) x^{j},
\end{aligned}
$$

which proves (44).

\section{The computation of the COEFFicients}

We take $\alpha$ real and $\alpha>-1$.

If we substitute $y(x)=L_{n}^{\alpha, M, N}(x)$ in the differential equation (4) and use (1) and (32), we find that 


$$
\begin{aligned}
M A_{0} & \sum_{i=0}^{\infty} a_{i}(x) D^{i} L_{n}^{(\alpha)}(x)+M A_{1} \sum_{i=0}^{\infty} a_{i}(x) D^{i+1} L_{n}^{(\alpha)}(x) \\
& +M A_{2} \sum_{i=0}^{\infty} a_{i}(x) D^{i+2} L_{n}^{(\alpha)}(x) \\
& +N A_{0} \sum_{i=0}^{\infty} b_{i}(x) D^{i} L_{n}^{(\alpha)}(x)+N A_{1} \sum_{i=0}^{\infty} b_{i}(x) D^{i+1} L_{n}^{(\alpha)}(x) \\
& +N A_{2} \sum_{i=0}^{\infty} b_{i}(x) D^{i+2} L_{n}^{(\alpha)}(x) \\
& +M N A_{0} \sum_{i=0}^{\infty} c_{i}(x) D^{i} L_{n}^{(\alpha)}(x)+M N A_{1} \sum_{i=0}^{\infty} c_{i}(x) D^{i+1} L_{n}^{(\alpha)}(x) \\
& +M N A_{2} \sum_{i=0}^{\infty} c_{i}(x) D^{i+2} L_{n}^{(\alpha)}(x) \\
& +A_{1}\left[x D^{3} L_{n}^{(\alpha)}(x)+(\alpha+1-x) D^{2} L_{n}^{(\alpha)}(x)+n D L_{n}^{(\alpha)}(x)\right] \\
+ & A_{2}\left[x D^{4} L_{n}^{(\alpha)}(x)+(\alpha+1-x) D^{3} L_{n}^{(\alpha)}(x)+n D^{2} L_{n}^{(\alpha)}(x)\right]=0 \\
& n=0,1,2, \ldots
\end{aligned}
$$

If we interpret the left-hand side as a polynomial in $M$ and $N$ and we use

$$
\begin{aligned}
x D^{3} L_{n}^{(\alpha)}(x)+(\alpha+1-x) D^{2} L_{n}^{(\alpha)}(x)+n D L_{n}^{(\alpha)}(x) & =D L_{n}^{(\alpha)}(x)-D^{2} L_{n}^{(\alpha)}(x) \\
& =-D^{2} L_{n+1}^{(\alpha)}(x)
\end{aligned}
$$

and

$$
\begin{aligned}
x D^{4} L_{n}^{(\alpha)}(x)+(\alpha+1-x) D^{3} L_{n}^{(\alpha)}(x)+n D^{2} L_{n}^{(\alpha)}(x) & =2 D^{2} L_{n}^{(\alpha)}(x)-2 D^{3} L_{n}^{(\alpha)}(x) \\
& =-2 D^{3} L_{n+1}^{(\alpha)}(x),
\end{aligned}
$$

which follow from the differential equation (32) for the classical Laguerre polynomials and (35), and the definition (2) of the coefficients $A_{0}, A_{1}$ and $A_{2}$, we find eight systems of equations for the coefficients $\left\{a_{i}(x)\right\}_{i=0}^{\infty},\left\{b_{i}(x)\right\}_{i=0}^{\infty}$ and $\left\{c_{i}(x)\right\}_{i=0}^{\infty}$. These can be written as follows:

$$
\begin{array}{llllll}
M & : & S_{1}=0 & N & : & S_{5}=0 \\
M^{2} & : & S_{2}=0 & N^{2} & : & S_{6}=0 \\
M N & : & S_{3}=0 & M N^{2} & : & S_{7}=0 \\
M^{2} N & : & S_{4}=0 & M^{2} N^{2} & : & S_{8}=0
\end{array}
$$

for $n=0,1,2, \ldots$, where

$$
S_{1}:=\sum_{i=0}^{\infty} a_{i}(x) D^{i} L_{n}^{(\alpha)}(x)-\left(\begin{array}{c}
n+\alpha \\
n
\end{array}\right) D^{2} L_{n+1}^{(\alpha)}(x),
$$




$$
\begin{aligned}
& S_{2}:=\left(\begin{array}{c}
n+\alpha \\
n-1
\end{array}\right) \sum_{i=0}^{\infty} a_{i}(x) D^{i} L_{n}^{(\alpha)}(x)+\left(\begin{array}{c}
n+\alpha \\
n
\end{array}\right) \sum_{i=0}^{\infty} a_{i}(x) D^{i+1} L_{n}^{(\alpha)}(x), \\
& S_{3}:=\frac{n(\alpha+2)-(\alpha+1)}{(\alpha+1)(\alpha+3)}\left(\begin{array}{c}
n+\alpha \\
n-2
\end{array}\right) \sum_{i=0}^{\infty} a_{i}(x) D^{i} L_{n}^{(\alpha)}(x) \\
& +\frac{n-1}{\alpha+1}\left(\begin{array}{c}
n+\alpha \\
n-1
\end{array}\right) \sum_{i=0}^{\infty} a_{i}(x) D^{i+1} L_{n}^{(\alpha)}(x) \\
& +\frac{1}{\alpha+1}\left(\begin{array}{c}
n+\alpha \\
n-1
\end{array}\right) \sum_{i=0}^{\infty} a_{i}(x) D^{i+2} L_{n}^{(\alpha)}(x)+\left(\begin{array}{c}
n+\alpha \\
n-1
\end{array}\right) \sum_{i=0}^{\infty} b_{i}(x) D^{i} L_{n}^{(\alpha)}(x) \\
& +\left(\begin{array}{c}
n+\alpha \\
n
\end{array}\right) \sum_{i=0}^{\infty} b_{i}(x) D^{i+1} L_{n}^{(\alpha)}(x)+\sum_{i=0}^{\infty} c_{i}(x) D^{i} L_{n}^{(\alpha)}(x) \\
& -\frac{2}{(\alpha+1)^{2}}\left(\begin{array}{c}
n+\alpha \\
n
\end{array}\right)\left[\left(\begin{array}{c}
n+\alpha+1 \\
n-2
\end{array}\right) D^{2} L_{n+1}^{(\alpha)}(x)+\left(\begin{array}{c}
n+\alpha+1 \\
n-1
\end{array}\right) D^{3} L_{n+1}^{(\alpha)}(x)\right], \\
& S_{4}:=\frac{1}{(\alpha+1)(\alpha+2)}\left(\begin{array}{c}
n+\alpha \\
n-1
\end{array}\right)\left(\begin{array}{c}
n+\alpha+1 \\
n-2
\end{array}\right) \sum_{i=0}^{\infty} a_{i}(x) D^{i} L_{n}^{(\alpha)}(x) \\
& +\frac{2}{(\alpha+1)^{2}}\left(\begin{array}{c}
n+\alpha \\
n
\end{array}\right)\left(\begin{array}{c}
n+\alpha+1 \\
n-2
\end{array}\right) \sum_{i=0}^{\infty} a_{i}(x) D^{i+1} L_{n}^{(\alpha)}(x) \\
& +\frac{1}{(\alpha+1)^{2}}\left(\begin{array}{c}
n+\alpha \\
n
\end{array}\right)\left(\begin{array}{c}
n+\alpha+1 \\
n-1
\end{array}\right) \sum_{i=0}^{\infty} a_{i}(x) D^{i+2} L_{n}^{(\alpha)}(x) \\
& +\left(\begin{array}{c}
n+\alpha \\
n-1
\end{array}\right) \sum_{i=0}^{\infty} c_{i}(x) D^{i} L_{n}^{(\alpha)}(x)+\left(\begin{array}{c}
n+\alpha \\
n
\end{array}\right) \sum_{i=0}^{\infty} c_{i}(x) D^{i+1} L_{n}^{(\alpha)}(x), \\
& S_{5}:=\sum_{i=0}^{\infty} b_{i}(x) D^{i} L_{n}^{(\alpha)}(x)-\frac{1}{\alpha+1}\left(\begin{array}{c}
n+\alpha \\
n-1
\end{array}\right)\left[(n-1) D^{2} L_{n+1}^{(\alpha)}(x)+2 D^{3} L_{n+1}^{(\alpha)}(x)\right], \\
& S_{6}:=\frac{n(\alpha+2)-(\alpha+1)}{(\alpha+1)(\alpha+3)}\left(\begin{array}{c}
n+\alpha \\
n-2
\end{array}\right) \sum_{i=0}^{\infty} b_{i}(x) D^{i} L_{n}^{(\alpha)}(x) \\
& +\frac{n-1}{\alpha+1}\left(\begin{array}{c}
n+\alpha \\
n-1
\end{array}\right) \sum_{i=0}^{\infty} b_{i}(x) D^{i+1} L_{n}^{(\alpha)}(x) \\
& +\frac{1}{\alpha+1}\left(\begin{array}{c}
n+\alpha \\
n-1
\end{array}\right) \sum_{i=0}^{\infty} b_{i}(x) D^{i+2} L_{n}^{(\alpha)}(x),
\end{aligned}
$$




$$
\begin{aligned}
S_{7}:=\frac{1}{(\alpha+1)(\alpha+2)}\left(\begin{array}{c}
n+\alpha \\
n-1
\end{array}\right)\left(\begin{array}{c}
n+\alpha+1 \\
n-2
\end{array}\right) \sum_{i=0}^{\infty} b_{i}(x) D^{i} L_{n}^{(\alpha)}(x) \\
+\frac{2}{(\alpha+1)^{2}}\left(\begin{array}{c}
n+\alpha \\
n
\end{array}\right)\left(\begin{array}{c}
n+\alpha+1 \\
n-2
\end{array}\right) \sum_{i=0}^{\infty} b_{i}(x) D^{i+1} L_{n}^{(\alpha)}(x) \\
+\frac{1}{(\alpha+1)^{2}}\left(\begin{array}{c}
n+\alpha \\
n
\end{array}\right)\left(\begin{array}{c}
n+\alpha+1 \\
n-1
\end{array}\right) \sum_{i=0}^{\infty} b_{i}(x) D^{i+2} L_{n}^{(\alpha)}(x) \\
+\frac{n(\alpha+2)-(\alpha+1)}{(\alpha+1)(\alpha+3)}\left(\begin{array}{c}
n+\alpha \\
n-2
\end{array}\right) \sum_{i=0}^{\infty} c_{i}(x) D^{i} L_{n}^{(\alpha)}(x) \\
+\frac{n-1}{\alpha+1}\left(\begin{array}{c}
n+\alpha \\
n-1
\end{array}\right) \sum_{i=0}^{\infty} c_{i}(x) D^{i+1} L_{n}^{(\alpha)}(x) \\
+\frac{1}{\alpha+1}\left(\begin{array}{c}
n+\alpha \\
n-1
\end{array}\right) \sum_{i=0}^{\infty} c_{i}(x) D^{i+2} L_{n}^{(\alpha)}(x)
\end{aligned}
$$

and

$$
\begin{aligned}
S_{8}:=\frac{1}{(\alpha+1)(\alpha+2)}\left(\begin{array}{c}
n+\alpha \\
n-1
\end{array}\right)\left(\begin{array}{c}
n+\alpha+1 \\
n-2
\end{array}\right) \sum_{i=0}^{\infty} c_{i}(x) D^{i} L_{n}^{(\alpha)}(x) \\
+\frac{2}{(\alpha+1)^{2}}\left(\begin{array}{c}
n+\alpha \\
n
\end{array}\right)\left(\begin{array}{c}
n+\alpha+1 \\
n-2
\end{array}\right) \sum_{i=0}^{\infty} c_{i}(x) D^{i+1} L_{n}^{(\alpha)}(x) \\
+\frac{1}{(\alpha+1)^{2}}\left(\begin{array}{c}
n+\alpha \\
n
\end{array}\right)\left(\begin{array}{c}
n+\alpha+1 \\
n-1
\end{array}\right) \sum_{i=0}^{\infty} c_{i}(x) D^{i+2} L_{n}^{(\alpha)}(x) .
\end{aligned}
$$

The systems of equations $S_{1}=0$ and $S_{2}=0$ for $n=0,1,2, \ldots$ lead to the solution for the coefficients $\left\{a_{i}(x)\right\}_{i=0}^{\infty}$ which was already found in [8]. The systems of equations $S_{5}=0$ and $S_{6}=0$ for $n=0,1,2, \ldots$ will lead to the solution for the coefficients $\left\{b_{i}(x)\right\}_{i=0}^{\infty}$ while the other four will eventually lead to the solution for the coefficients $\left\{c_{i}(x)\right\}_{i=0}^{\infty}$.

4.1. The computation of the coefficients $\left\{a_{i}(x)\right\}_{i=0}^{\infty}$. Since $\alpha>-1$ we have $\left(\begin{array}{c}n+\alpha \\ n\end{array}\right) \neq 0$ for all $n=0,1,2, \ldots$ Then the systems of equations $S_{1}=0$ and $S_{2}=0$ for $n=0,1,2, \ldots$ are equivalent to

$$
\sum_{i=0}^{\infty} a_{i}(x) D^{i} L_{n}^{(\alpha)}(x)=\left(\begin{array}{c}
n+\alpha \\
n
\end{array}\right) D^{2} L_{n+1}^{(\alpha)}(x), n=0,1,2, \ldots,
$$

and

$$
\sum_{i=0}^{\infty} a_{i}(x) D^{i+1} L_{n}^{(\alpha)}(x)=-\left(\begin{array}{c}
n+\alpha \\
n-1
\end{array}\right) D^{2} L_{n+1}^{(\alpha)}(x), n=0,1,2, \ldots .
$$

By considering equation (45) for $n=0$ and equation (46) for $n=0$ and $n=1$ we conclude that $a_{0}(0, \alpha)=0$ and $a_{0}(1, \alpha)=1$. Since $a_{i}(x)$ must be a polynomial in $x$ of degree at most $i$ for each $i=1,2,3, \ldots$, we may write

$$
a_{i}(x)=k_{i} x^{i}+\text { lower order terms, } i=1,2,3, \ldots .
$$


By comparing the coefficients of highest degree in (45) and (46) we find that

$$
\frac{a_{0}(n, \alpha)}{n !}+\sum_{i=1}^{n} \frac{k_{i}}{(n-i) !}=0, n=1,2,3, \ldots
$$

and

$$
\frac{a_{0}(n, \alpha)}{(n-1) !}+\sum_{i=1}^{n-1} \frac{k_{i}}{(n-i-1) !}=\left(\begin{array}{c}
n+\alpha \\
n-1
\end{array}\right) \frac{1}{(n-1) !}, n=2,3,4, \ldots
$$

Hence, since $k_{i}$ is independent of $n$, we obtain

$$
a_{0}(n, \alpha)-a_{0}(n-1, \alpha)=\left(\begin{array}{c}
n+\alpha \\
n-1
\end{array}\right), n=1,2,3, \ldots,
$$

and therefore, by using (25)

$$
a_{0}(n, \alpha)=a_{0}(n, \alpha)-a_{0}(0, \alpha)=\sum_{k=1}^{n}\left(\begin{array}{c}
k+\alpha \\
k-1
\end{array}\right)=\left(\begin{array}{c}
n+\alpha+1 \\
n-1
\end{array}\right), n=1,2,3, \ldots,
$$

which proves (5). Note that this proof of $(5)$ is different from the one given in [1].

In order to prove (6) we write, instead of (45) and (46),

$$
\sum_{i=1}^{\infty} a_{i}(x) D^{i} L_{n}^{(\alpha)}(x)=\left(\begin{array}{c}
n+\alpha \\
n
\end{array}\right) D^{2} L_{n+1}^{(\alpha)}(x)-a_{0}(n, \alpha) L_{n}^{(\alpha)}(x), n=0,1,2, \ldots
$$

and

$$
\begin{aligned}
\sum_{i=1}^{\infty} a_{i}(x) D^{i+1} L_{n}^{(\alpha)}(x) \\
\quad=-\left(\begin{array}{c}
n+\alpha \\
n-1
\end{array}\right) D^{2} L_{n+1}^{(\alpha)}(x)-a_{0}(n, \alpha) D L_{n}^{(\alpha)}(x), n=0,1,2, \ldots
\end{aligned}
$$

First we will prove that every solution of (48) is also a solution of (47). Note that

$$
a_{0}(n+1, \alpha)=a_{0}(n, \alpha)+\left(\begin{array}{c}
n+\alpha+1 \\
n
\end{array}\right), n=0,1,2, \ldots,
$$


which was already obtained before. Now suppose that $\left\{a_{i}(x)\right\}_{i=1}^{\infty}$ is a solution of (48). Then we use (34), (35) and (49) to find for the left-hand side of (47)

$$
\begin{aligned}
\sum_{i=1}^{\infty} a_{i}(x) D^{i} L_{n}^{(\alpha)}(x) & \\
= & \sum_{i=1}^{\infty} a_{i}(x) D^{i+1} L_{n}^{(\alpha)}(x)-\sum_{i=1}^{\infty} a_{i}(x) D^{i+1} L_{n+1}^{(\alpha)}(x) \\
= & \left(\begin{array}{c}
n+\alpha+1 \\
n
\end{array}\right) D^{2} L_{n+2}^{(\alpha)}(x)-\left(\begin{array}{c}
n+\alpha \\
n-1
\end{array}\right) D^{2} L_{n+1}^{(\alpha)}(x) \\
& +a_{0}(n+1, \alpha) D L_{n+1}^{(\alpha)}(x)-a_{0}(n, \alpha) D L_{n}^{(\alpha)}(x) \\
= & \left(\begin{array}{c}
n+\alpha+1 \\
n
\end{array}\right)\left[D^{2} L_{n+1}^{(\alpha)}(x)-D L_{n+1}^{(\alpha)}(x)\right]-\left(\begin{array}{c}
n+\alpha \\
n-1
\end{array}\right) D^{2} L_{n+1}^{(\alpha)}(x) \\
+ & {\left.\left[\begin{array}{c}
n \\
\left.a_{0}(n, \alpha)+\left(\begin{array}{c}
n+\alpha+1 \\
n
\end{array}\right)\right] D L_{n+1}^{(\alpha)}(x)-a_{0}(n, \alpha) D L_{n}^{(\alpha)}(x) \\
n+\alpha+1
\end{array}\right)-\left(\begin{array}{c}
n+\alpha \\
n-1
\end{array}\right)\right] D^{2} L_{n+1}^{(\alpha)}(x)+a_{0}(n, \alpha)\left[D L_{n+1}^{(\alpha)}(x)-D L_{n}^{(\alpha)}(x)\right] } \\
= & \left(\begin{array}{c}
n+\alpha \\
n
\end{array}\right) D^{2} L_{n+1}^{(\alpha)}(x)-a_{0}(n, \alpha) L_{n}^{(\alpha)}(x), n=0,1,2, \ldots,
\end{aligned}
$$

which equals the right-hand side of (47).

Now we will solve (48). We have by using (5), (26) and (31)

$$
\begin{aligned}
& -\left(\begin{array}{c}
n+\alpha \\
n-1
\end{array}\right) D^{2} L_{n+1}^{(\alpha)}(x)-a_{0}(n, \alpha) D L_{n}^{(\alpha)}(x) \\
& =\left(\begin{array}{c}
n+\alpha+1 \\
n-1
\end{array}\right) L_{n-1}^{(\alpha+1)}(x)-\left(\begin{array}{c}
n+\alpha \\
n-1
\end{array}\right) L_{n-1}^{(\alpha+2)}(x) \\
& =-\frac{(n-1) x}{(\alpha+2)(\alpha+3)}\left(\begin{array}{c}
n+\alpha \\
n-1
\end{array}\right)\left(\begin{array}{c}
n+\alpha+1 \\
n-1
\end{array}\right) \sum_{k=0}^{\infty} \frac{(-n+2)_{k}}{(\alpha+4)_{k}} \frac{x^{k}}{k !} \\
& =-\frac{x}{\alpha+2}\left(\begin{array}{c}
n+\alpha \\
n-1
\end{array}\right) L_{n-2}^{(\alpha+3)}(x), n=2,3,4, \ldots
\end{aligned}
$$

Note that the right-hand side of (48) equals zero for $n=0$ and $n=1$, which is necessary for solvability. Hence, the system of equations (48) is equivalent to

$$
\sum_{i=1}^{\infty} a_{i}(x) D^{i+1} L_{n}^{(\alpha)}(x)=-\frac{x}{\alpha+2}\left(\begin{array}{c}
n+\alpha \\
n-1
\end{array}\right) L_{n-2}^{(\alpha+3)}(x), n=2,3,4, \ldots
$$

Now we apply the inversion formula of Lemma 5 to obtain

$$
a_{i}(x)=\frac{(-1)^{i} x}{\alpha+2} \sum_{j=1}^{i}\left(\begin{array}{c}
j+\alpha+1 \\
j
\end{array}\right) L_{i-j}^{(-\alpha-i-2)}(-x) L_{j-1}^{(\alpha+3)}(x), i=1,2,3, \ldots,
$$

and with (37) and (40) we obtain

$$
\begin{aligned}
a_{i}(x) & =(-1)^{i} x F_{i-1}(\alpha+3, \alpha+3 ; 2 ; x) \\
& =\frac{1}{i !} \sum_{j=1}^{i}(-1)^{i+j+1}\left(\begin{array}{c}
\alpha+1 \\
j-1
\end{array}\right)\left(\begin{array}{c}
\alpha+2 \\
i-j
\end{array}\right)(\alpha+3)_{i-j} x^{j}, i=1,2,3, \ldots,
\end{aligned}
$$

which equals (6). 
4.2. The computation of the coefficients $\left\{b_{i}(x)\right\}_{i=0}^{\infty}$. We use the systems of equations $S_{5}=0$ and $S_{6}=0$ for $n=0,1,2, \ldots$ to find the coefficients $\left\{b_{i}(x)\right\}_{i=0}^{\infty}$. Since $\alpha>-1$ we have $\left(\begin{array}{l}n+\alpha \\ n-1\end{array}\right) \neq 0$ for $n=1,2,3, \ldots$ We use this to conclude that these systems are equivalent to

$$
\sum_{i=0}^{\infty} b_{i}(x) D^{i} L_{n}^{(\alpha)}(x)=\frac{1}{\alpha+1}\left(\begin{array}{c}
n+\alpha \\
n-1
\end{array}\right)\left[(n-1) D^{2} L_{n+1}^{(\alpha)}(x)+2 D^{3} L_{n+1}^{(\alpha)}(x)\right]
$$

for $n=0,1,2, \ldots$ and

$$
\begin{aligned}
& (n-1) \sum_{i=0}^{\infty} b_{i}(x) D^{i+1} L_{n}^{(\alpha)}(x)+\sum_{i=0}^{\infty} b_{i}(x) D^{i+2} L_{n}^{(\alpha)}(x) \\
& =-\frac{n(\alpha+2)-(\alpha+1)}{(\alpha+1)(\alpha+3)}\left(\begin{array}{c}
n+\alpha \\
n-2
\end{array}\right)\left[(n-1) D^{2} L_{n+1}^{(\alpha)}(x)+2 D^{3} L_{n+1}^{(\alpha)}(x)\right]
\end{aligned}
$$

for $n=1,2,3, \ldots$ For $n=0$ equation (50) leads to $b_{0}(0, \alpha)=0$, and for $n=1$ equation (51) is trivial. Also, we conclude that $b_{0}(1, \alpha)$ is arbitrary. Since $b_{i}(x)$ must be a polynomial in $x$ of degree at most $i$ for each $i=1,2,3, \ldots$, we may write

$$
b_{i}(x)=k_{i}^{\prime} x^{i}+\text { lower order terms, } i=1,2,3, \ldots .
$$

Then we find, by comparing the coefficients of highest degree in (50) and (51), that

$$
\frac{b_{0}(n, \alpha)}{n !}+\sum_{i=1}^{n} \frac{k_{i}^{\prime}}{(n-i) !}=0, n=1,2,3, \ldots,
$$

and

$$
\begin{aligned}
& \frac{b_{0}(n, \alpha)}{(n-1) !}+\sum_{i=1}^{n-1} \frac{k_{i}^{\prime}}{(n-i-1) !} \\
& \quad=\frac{n(\alpha+2)-(\alpha+1)}{(\alpha+1)(\alpha+3)}\left(\begin{array}{c}
n+\alpha \\
n-2
\end{array}\right) \frac{1}{(n-1) !}, n=2,3,4, \ldots .
\end{aligned}
$$

Hence, since $k_{i}^{\prime}$ is independent of $n$, we obtain

$$
b_{0}(n, \alpha)-b_{0}(n-1, \alpha)=\frac{n(\alpha+2)-(\alpha+1)}{(\alpha+1)(\alpha+3)}\left(\begin{array}{c}
n+\alpha \\
n-2
\end{array}\right), n=2,3,4, \ldots,
$$

and therefore, by using (25),

$$
\begin{aligned}
b_{0}(n, \alpha)-b_{0}(1, \alpha) & =\sum_{k=2}^{n} \frac{k(\alpha+2)-(\alpha+1)}{(\alpha+1)(\alpha+3)}\left(\begin{array}{c}
k+\alpha \\
k-2
\end{array}\right) \\
& =\frac{\alpha+2}{\alpha+1} \sum_{k=2}^{n}\left(\begin{array}{c}
k+\alpha \\
k-3
\end{array}\right)+\frac{1}{\alpha+1} \sum_{k=2}^{n}\left(\begin{array}{c}
k+\alpha \\
k-2
\end{array}\right) \\
& =\frac{\alpha+2}{\alpha+1}\left(\begin{array}{c}
n+\alpha+1 \\
n-3
\end{array}\right)+\frac{1}{\alpha+1}\left(\begin{array}{c}
n+\alpha+1 \\
n-2
\end{array}\right) \\
& =\frac{n(\alpha+2)-\alpha}{(\alpha+1)(\alpha+4)}\left(\begin{array}{c}
n+\alpha+1 \\
n-2
\end{array}\right), n=1,2,3, \ldots
\end{aligned}
$$

This proves that $b_{0}(n, \alpha)-b_{0}(1, \alpha)=\beta_{0}(n, \alpha), n=1,2,3, \ldots$, where $\beta_{0}(n, \alpha)$ is given by (11). 
Now we will show that every solution of (50) also satisfies (51). In order to do this we write (50) in the form

$$
\sum_{i=1}^{\infty} b_{i}(x) D^{i} L_{n}^{(\alpha)}(x)=F_{n}(x), n=0,1,2, \ldots
$$

where

$$
F_{n}(x)=\frac{1}{\alpha+1}\left(\begin{array}{c}
n+\alpha \\
n-1
\end{array}\right)\left[(n-1) D^{2} L_{n+1}^{(\alpha)}(x)+2 D^{3} L_{n+1}^{(\alpha)}(x)\right]-b_{0}(n, \alpha) L_{n}^{(\alpha)}(x) .
$$

Suppose that $\left\{b_{i}(x)\right\}_{i=1}^{\infty}$ is a solution of (54). If we now write

$$
\sum_{i=1}^{\infty} b_{i}(x) D^{i+1} L_{n}^{(\alpha)}(x)=G_{n}(x), n=0,1,2, \ldots,
$$

then we find by using (34) that

$$
G_{n}(x)-G_{n+1}(x)=\sum_{i=1}^{\infty} b_{i}(x) D^{i} L_{n}^{(\alpha)}(x)=F_{n}(x), n=0,1,2, \ldots
$$

Hence, we have $G_{0}(x)=0$ and

$$
G_{n}(x)=-\sum_{k=0}^{n-1} F_{k}(x), n=1,2,3, \ldots
$$

In a similar way we find that

$$
\begin{aligned}
\sum_{i=1}^{\infty} b_{i}(x) D^{i+2} L_{n}^{(\alpha)}(x) & =-\sum_{m=0}^{n-1} G_{m}(x)=\sum_{m=1}^{n-1} \sum_{k=0}^{m-1} F_{k}(x) \\
& =\sum_{k=0}^{n-2} \sum_{m=k+1}^{n-1} F_{k}(x)=\sum_{k=0}^{n-2}(n-1-k) F_{k}(x) \\
& =(n-1) \sum_{k=0}^{n-1} F_{k}(x)-\sum_{k=0}^{n-1} k F_{k}(x), n=2,3,4, \ldots
\end{aligned}
$$

Hence

$$
\begin{gathered}
(n-1) \sum_{i=1}^{\infty} b_{i}(x) D^{i+1} L_{n}^{(\alpha)}(x)+\sum_{i=1}^{\infty} b_{i}(x) D^{i+2} L_{n}^{(\alpha)}(x) \\
=-\sum_{k=0}^{n-1} k F_{k}(x), n=1,2,3, \ldots
\end{gathered}
$$

In view of (51) we have to prove that

$$
\begin{aligned}
& \sum_{k=0}^{n-1} k F_{k}(x)=\frac{n(\alpha+2)-(\alpha+1)}{(\alpha+1)(\alpha+3)}\left(\begin{array}{c}
n+\alpha \\
n-2
\end{array}\right)\left[(n-1) D^{2} L_{n+1}^{(\alpha)}(x)+2 D^{3} L_{n+1}^{(\alpha)}(x)\right] \\
& \quad+b_{0}(n, \alpha)\left[(n-1) D L_{n}^{(\alpha)}(x)+D^{2} L_{n}^{(\alpha)}(x)\right], n=1,2,3, \ldots
\end{aligned}
$$


This is done by induction. For $n=1$ this formula is trivial. Suppose that (55) holds for certain $n \in\{1,2,3, \ldots\}$. Then we have to show that

$$
\begin{gathered}
\sum_{k=0}^{n} k F_{k}(x)=\frac{n(\alpha+2)+1}{(\alpha+1)(\alpha+3)}\left(\begin{array}{c}
n+\alpha+1 \\
n-1
\end{array}\right)\left[n D^{2} L_{n+2}^{(\alpha)}(x)+2 D^{3} L_{n+2}^{(\alpha)}(x)\right] \\
+b_{0}(n+1, \alpha)\left[n D L_{n+1}^{(\alpha)}(x)+D^{2} L_{n+1}^{(\alpha)}(x)\right] .
\end{gathered}
$$

We find that

$$
\begin{aligned}
& \sum_{k=0}^{n} k F_{k}(x)=n F_{n}(x)+\sum_{k=0}^{n-1} k F_{k}(x) \\
& =n F_{n}(x)+\frac{n(\alpha+2)-(\alpha+1)}{(\alpha+1)(\alpha+3)}\left(\begin{array}{c}
n+\alpha \\
n-2
\end{array}\right)\left[(n-1) D^{2} L_{n+1}^{(\alpha)}(x)+2 D^{3} L_{n+1}^{(\alpha)}(x)\right] \\
& \quad+b_{0}(n, \alpha)\left[(n-1) D L_{n}^{(\alpha)}(x)+D^{2} L_{n}^{(\alpha)}(x)\right]
\end{aligned}
$$

Earlier we have found that

(56)

$$
b_{0}(n+1, \alpha)=b_{0}(n, \alpha)+\frac{n(\alpha+2)+1}{(\alpha+1)(\alpha+3)}\left(\begin{array}{c}
n+\alpha+1 \\
n-1
\end{array}\right), n=1,2,3, \ldots
$$

We also have

$$
\frac{n}{\alpha+1}\left(\begin{array}{c}
n+\alpha \\
n-1
\end{array}\right)+\frac{n(\alpha+2)-(\alpha+1)}{(\alpha+1)(\alpha+3)}\left(\begin{array}{c}
n+\alpha \\
n-2
\end{array}\right)=\frac{n(\alpha+2)+1}{(\alpha+1)(\alpha+3)}\left(\begin{array}{c}
n+\alpha+1 \\
n-1
\end{array}\right) .
$$

Hence we find by using (35) that

$$
\begin{aligned}
& \sum_{k=0}^{n} k F_{k}(x)=\frac{n(\alpha+2)+1}{(\alpha+1)(\alpha+3)}\left(\begin{array}{c}
n+\alpha+1 \\
n-1
\end{array}\right) \\
& \times\left[(n-1) D^{2} L_{n+1}^{(\alpha)}(x)+2 D^{3} L_{n+1}^{(\alpha)}(x)-n D L_{n+1}^{(\alpha)}(x)-D^{2} L_{n+1}^{(\alpha)}(x)\right] \\
& +b_{0}(n+1, \alpha)\left[n D L_{n+1}^{(\alpha)}(x)+D^{2} L_{n+1}^{(\alpha)}(x)\right] \\
& =\frac{n(\alpha+2)+1}{(\alpha+1)(\alpha+3)}\left(\begin{array}{c}
n+\alpha+1 \\
n-1
\end{array}\right)\left[n D^{2} L_{n+2}^{(\alpha)}(x)+2 D^{3} L_{n+2}^{(\alpha)}(x)\right] \\
& +b_{0}(n+1, \alpha)\left[n D L_{n+1}^{(\alpha)}(x)+D^{2} L_{n+1}^{(\alpha)}(x)\right] .
\end{aligned}
$$

This completes the proof.

Now we will solve (54). Note that $F_{0}(x)=0$, which is necessary for solvability. Substitution of $b_{0}(n, \alpha)=b_{0}(1, \alpha)+\beta_{0}(n, \alpha), n=1,2,3, \ldots$, into the right-hand side of (54) now leads to

$$
F_{n}(x)=-b_{0}(1, \alpha) L_{n}^{(\alpha)}(x)+H_{n}(x), n=1,2,3, \ldots,
$$


where

$$
\begin{array}{r}
H_{n}(x)=\frac{1}{\alpha+1}\left(\begin{array}{c}
n+\alpha \\
n-1
\end{array}\right)\left[(n-1) D^{2} L_{n+1}^{(\alpha)}(x)+2 D^{3} L_{n+1}^{(\alpha)}(x)\right]- \\
-\beta_{0}(n, \alpha) L_{n}^{(\alpha)}(x), \\
n=1,2,3, \ldots .
\end{array}
$$

As before, we have

$$
\begin{aligned}
\beta_{0}(n, \alpha) & =\frac{n(\alpha+2)-\alpha}{(\alpha+1)(\alpha+4)}\left(\begin{array}{c}
n+\alpha+1 \\
n-2
\end{array}\right) \\
& =\frac{1}{\alpha+1}\left(\begin{array}{c}
n+\alpha+1 \\
n-2
\end{array}\right)+\frac{\alpha+2}{\alpha+1}\left(\begin{array}{c}
n+\alpha+1 \\
n-3
\end{array}\right), n=1,2,3, \ldots .
\end{aligned}
$$

Hence, since $\beta_{0}(1, \alpha)=0$, we find that $H_{1}(x)=0$. Further, by (31)

$$
\begin{aligned}
& H_{n}(x)=\frac{\alpha+2}{\alpha+1}\left(\begin{array}{c}
n+\alpha \\
n-2
\end{array}\right) L_{n-1}^{(\alpha+2)}(x)-\frac{2}{\alpha+1}\left(\begin{array}{c}
n+\alpha \\
n-1
\end{array}\right) L_{n-2}^{(\alpha+3)}(x) \\
& -\frac{1}{\alpha+1}\left(\begin{array}{c}
n+\alpha+1 \\
n-2
\end{array}\right) L_{n}^{(\alpha)}(x)-\frac{\alpha+2}{\alpha+1}\left(\begin{array}{c}
n+\alpha+1 \\
n-3
\end{array}\right) L_{n}^{(\alpha)}(x), n=2,3,4, \ldots .
\end{aligned}
$$

Applying the inversion formula of Lemma 5, we obtain

$$
b_{i}(x)=b_{0}(1, \alpha) b_{i}^{*}(x)+\beta_{i}(x), i=1,2,3, \ldots,
$$

where, by using (36) and (28),

$$
\begin{aligned}
b_{i}^{*}(x) & =(-1)^{i+1} \sum_{j=1}^{i} L_{i-j}^{(-\alpha-i-1)}(-x) L_{j}^{(\alpha)}(x)=(-1)^{i} L_{i}^{(-\alpha-i-1)}(-x) \\
& =\sum_{j=0}^{i} \frac{(\alpha+1)_{i-j}}{(i-j) !} \frac{(-x)^{j}}{j !}=\frac{1}{i !} \sum_{j=0}^{i}(-1)^{j}\left(\begin{array}{c}
i \\
j
\end{array}\right)(\alpha+1)_{i-j} x^{j}, i=1,2,3, \ldots,
\end{aligned}
$$

which equals (9). Further we obtain that $\beta_{1}(x)=0$ and

$$
\beta_{i}(x):=\beta_{i}^{(1)}(x)+\beta_{i}^{(2)}(x)+\beta_{i}^{(3)}(x)+\beta_{i}^{(4)}(x), i=2,3,4, \ldots,
$$

where

$$
\begin{gathered}
\beta_{i}^{(1)}(x)=(-1)^{i} \frac{\alpha+2}{\alpha+1} \sum_{j=2}^{i}\left(\begin{array}{c}
j+\alpha \\
j-2
\end{array}\right) L_{i-j}^{(-\alpha-i-1)}(-x) L_{j-1}^{(\alpha+2)}(x), i=2,3,4, \ldots, \\
\beta_{i}^{(2)}(x)=\frac{2(-1)^{i+1}}{\alpha+1} \sum_{j=2}^{i}\left(\begin{array}{c}
j+\alpha \\
j-1
\end{array}\right) L_{i-j}^{(-\alpha-i-1)}(-x) L_{j-2}^{(\alpha+3)}(x), i=2,3,4, \ldots, \\
\beta_{i}^{(3)}(x)=\frac{(-1)^{i+1}}{\alpha+1} \sum_{j=2}^{i}\left(\begin{array}{c}
j+\alpha+1 \\
j-2
\end{array}\right) L_{i-j}^{(-\alpha-i-1)}(-x) L_{j}^{(\alpha)}(x), i=2,3,4, \ldots,
\end{gathered}
$$

and

$$
\beta_{i}^{(4)}(x)=(-1)^{i+1} \frac{\alpha+2}{\alpha+1} \sum_{j=2}^{i}\left(\begin{array}{c}
j+\alpha+1 \\
j-3
\end{array}\right) L_{i-j}^{(-\alpha-i-1)}(-x) L_{j}^{(\alpha)}(x), i=2,3,4, \ldots
$$

Note that by using (37) and (38) we have

$$
\beta_{i}^{(1)}(x)=\frac{(-1)^{i}}{\alpha+1} F_{i-1}(\alpha+2, \alpha+2 ; 0 ; x), i=2,3,4, \ldots,
$$




$$
\begin{aligned}
& \beta_{i}^{(2)}(x)=(-1)^{i+1} \frac{2(\alpha+2)}{\alpha+1} F_{i-2}(\alpha+3, \alpha+3 ; 2 ; x), i=2,3,4, \ldots \\
& \beta_{i}^{(3)}(x)=\frac{(-1)^{i+1}}{(\alpha+1)(\alpha+2)(\alpha+3)} G_{i}(\alpha+2, \alpha ;-1 ; x), i=2,3,4, \ldots
\end{aligned}
$$

and

$$
\beta_{i}^{(4)}(x)=\frac{(-1)^{i+1}}{(\alpha+1)(\alpha+3)(\alpha+4)} G_{i}(\alpha+2, \alpha ;-2 ; x), i=2,3,4, \ldots .
$$

Applying (40) and (41), we now easily obtain (13), (14), (15) and (16). We remark that

$$
x \beta_{i}^{(2)}(x)=\frac{2(\alpha+2)}{\alpha+1} a_{i-1}(x), i=2,3,4, \ldots
$$

4.3. The computation of the coefficients $\left\{c_{i}(x)\right\}_{i=0}^{\infty}$. We will use the systems of equations $S_{3}=0, S_{4}=0, S_{7}=0$ and $S_{8}=0$ for $n=0,1,2, \ldots$ to find the coefficients $\left\{c_{i}(x)\right\}_{i=0}^{\infty}$. For $n=0$ these equations lead to $c_{0}(0, \alpha)=0$.

First of all we use (51) to write

$$
\sum_{i=0}^{\infty} b_{i}(x) D^{i+2} L_{n}^{(\alpha)}(x)=-(n-1) \sum_{i=0}^{\infty} b_{i}(x) D^{i+1} L_{n}^{(\alpha)}(x)
$$

$$
-\frac{n(\alpha+2)-(\alpha+1)}{(\alpha+1)(\alpha+3)}\left(\begin{array}{c}
n+\alpha \\
n-2
\end{array}\right)\left[(n-1) D^{2} L_{n+1}^{(\alpha)}(x)+2 D^{3} L_{n+1}^{(\alpha)}(x)\right]
$$

for $n=0,1,2, \ldots$ Now we substitute (45), (46), (50) and (57) into the four systems of equations. Then we will show that

$$
(n-1)\left(\begin{array}{c}
n+\alpha+1 \\
n-1
\end{array}\right)\left[\left(\begin{array}{c}
n+\alpha+1 \\
n-1
\end{array}\right) S_{3}-n S_{4}\right]
$$

$$
+(\alpha+1)(\alpha+3)\left[\left(\begin{array}{c}
n+\alpha+1 \\
n-1
\end{array}\right) S_{7}-n S_{8}\right]=0, n=0,1,2, \ldots
$$

This can be done by straightforward but tedious computations as follows. First we obtain

$$
\begin{aligned}
& \left(\begin{array}{c}
n+\alpha+1 \\
n-1
\end{array}\right) S_{3}-n S_{4} \\
& =\left(\begin{array}{c}
n+\alpha \\
n
\end{array}\right)\left(\begin{array}{c}
n+\alpha+1 \\
n-1
\end{array}\right) \sum_{i=0}^{\infty} b_{i}(x) D^{i+1} L_{n}^{(\alpha)}(x) \\
& -(\alpha+1)\left(\begin{array}{c}
n+\alpha \\
n-2
\end{array}\right) \sum_{i=0}^{\infty} c_{i}(x) D^{i} L_{n}^{(\alpha)}(x)-n\left(\begin{array}{c}
n+\alpha \\
n
\end{array}\right) \sum_{i=0}^{\infty} c_{i}(x) D^{i+1} L_{n}^{(\alpha)}(x) \\
& +\frac{1}{\alpha+1}\left(\begin{array}{c}
n+\alpha \\
n-2
\end{array}\right)\left(\begin{array}{c}
n+\alpha \\
n
\end{array}\right)\left(\begin{array}{c}
n+\alpha+1 \\
n-1
\end{array}\right) \\
& \times\left[(n-1) D^{2} L_{n+1}^{(\alpha)}(x)+2 D^{3} L_{n+1}^{(\alpha)}(x)\right], n=0,1,2, \ldots,
\end{aligned}
$$


and

$$
\begin{aligned}
\left(\begin{array}{c}
n+\alpha+1 \\
n-1
\end{array}\right) S_{7}-n S_{8} \\
=-\frac{1}{\alpha+1}\left(\begin{array}{c}
n+\alpha \\
n
\end{array}\right)\left(\begin{array}{c}
n+\alpha+1 \\
n-2
\end{array}\right)\left(\begin{array}{c}
n+\alpha+1 \\
n-1
\end{array}\right) \sum_{i=0}^{\infty} b_{i}(x) D^{i+1} L_{n}^{(\alpha)}(x) \\
\quad+\left(\begin{array}{c}
n+\alpha \\
n-2
\end{array}\right)\left(\begin{array}{c}
n+\alpha+1 \\
n-2
\end{array}\right) \sum_{i=0}^{\infty} c_{i}(x) D^{i} L_{n}^{(\alpha)}(x) \\
\quad+\left(\begin{array}{c}
n+\alpha \\
n-1
\end{array}\right)\left(\begin{array}{c}
n+\alpha+1 \\
n-2
\end{array}\right) \sum_{i=0}^{\infty} c_{i}(x) D^{i+1} L_{n}^{(\alpha)}(x) \\
\quad-\frac{1}{(\alpha+1)^{2}}\left(\begin{array}{c}
n+\alpha \\
n-2
\end{array}\right)\left(\begin{array}{c}
n+\alpha \\
n
\end{array}\right)\left(\begin{array}{c}
n+\alpha+1 \\
n-2
\end{array}\right)\left(\begin{array}{c}
n+\alpha+1 \\
n-1
\end{array}\right) \\
\quad \times\left[\begin{array}{c}
\left.(n-1) D^{2} L_{n+1}^{(\alpha)}(x)+2 D^{3} L_{n+1}^{(\alpha)}(x)\right], n=0,1,2, \ldots,
\end{array}\right.
\end{aligned}
$$

which eventually lead to (58).

Now we use (58) to conclude that for $n=1,2,3, \ldots$ the systems of equations $S_{3}=0, S_{4}=0, S_{7}=0$ and $S_{8}=0$ are equivalent to

$$
S_{3}=0,\left(\begin{array}{c}
n+\alpha+1 \\
n-1
\end{array}\right) S_{3}-n S_{4}=0 \text { and } S_{8}=0 .
$$

Finally this leads to the following systems of equations:

$$
\begin{aligned}
& \begin{aligned}
\sum_{i=0}^{\infty} c_{i}(x) D^{i} L_{n}^{(\alpha)}(x) & \\
= & -\frac{n \alpha-(\alpha+1)}{(\alpha+1)^{2}}\left(\begin{array}{c}
n+\alpha \\
n-2
\end{array}\right)\left(\begin{array}{c}
n+\alpha \\
n
\end{array}\right) D^{2} L_{n+1}^{(\alpha)}(x) \\
& \quad-\frac{2}{\alpha+1}\left(\begin{array}{c}
n+\alpha \\
n-2
\end{array}\right)\left(\begin{array}{c}
n+\alpha \\
n
\end{array}\right) D^{3} L_{n+1}^{(\alpha)}(x) \\
& \quad-\frac{1}{\alpha+1}\left(\begin{array}{c}
n+\alpha \\
n-1
\end{array}\right) \sum_{i=0}^{\infty} a_{i}(x) D^{i+2} L_{n}^{(\alpha)}(x)-\left(\begin{array}{c}
n+\alpha \\
n
\end{array}\right) \sum_{i=0}^{\infty} b_{i}(x) D^{i+1} L_{n}^{(\alpha)}(x), \\
& \sum_{i=0}^{\infty} c_{i}(x) D^{i+1} L_{n}^{(\alpha)}(x) \\
= & \left(\begin{array}{c}
n+\alpha \\
n-2
\end{array}\right)^{2} D^{2} L_{n+1}^{(\alpha)}(x)+\frac{2}{\alpha+1}\left(\begin{array}{c}
n+\alpha \\
n-2
\end{array}\right)\left(\begin{array}{c}
n+\alpha \\
n-1
\end{array}\right) D^{3} L_{n+1}^{(\alpha)}(x)
\end{aligned}
\end{aligned}
$$

$$
+\frac{1}{\alpha+1}\left(\begin{array}{c}
n+\alpha \\
n-2
\end{array}\right) \sum_{i=0}^{\infty} a_{i}(x) D^{i+2} L_{n}^{(\alpha)}(x)+\left(\begin{array}{c}
n+\alpha \\
n-1
\end{array}\right) \sum_{i=0}^{\infty} b_{i}(x) D^{i+1} L_{n}^{(\alpha)}(x),
$$


and

$$
\begin{aligned}
& \sum_{i=0}^{\infty} c_{i}(x) D^{i+2} L_{n}^{(\alpha)}(x) \\
& =-\frac{n(\alpha+2)-(\alpha+1)}{(\alpha+1)(\alpha+3)}\left(\begin{array}{c}
n+\alpha \\
n-2
\end{array}\right)^{2} D^{2} L_{n+1}^{(\alpha)}(x)-\frac{2}{\alpha+1}\left(\begin{array}{c}
n+\alpha \\
n-2
\end{array}\right)^{2} D^{3} L_{n+1}^{(\alpha)}(x)
\end{aligned}
$$

$$
-\frac{1}{\alpha+1}\left(\begin{array}{c}
n+\alpha \\
n-3
\end{array}\right) \sum_{i=0}^{\infty} a_{i}(x) D^{i+2} L_{n}^{(\alpha)}(x)-\left(\begin{array}{c}
n+\alpha \\
n-2
\end{array}\right) \sum_{i=0}^{\infty} b_{i}(x) D^{i+1} L_{n}^{(\alpha)}(x)
$$

for $n=0,1,2, \ldots$ For $n=0$ we only find that $c_{0}(0, \alpha)=0$, and for $n=1$ we find that $c_{0}(1, \alpha)=b_{0}(1, \alpha)$ and $c_{1}(x)=-b_{0}(1, \alpha) x$. Since $c_{i}(x)$ must be a polynomial in $x$ of degree at most $i$ for each $i=1,2,3, \ldots$, we may write

$$
c_{i}(x)=k_{i}^{\prime \prime} x^{i}+\text { lower order terms, } i=1,2,3, \ldots \text {. }
$$

Then, by comparing the coefficients of highest degree in (59) and (60) by using (52), we find that

$$
\frac{c_{0}(n, \alpha)}{n !}+\sum_{i=1}^{n} \frac{k_{i}^{\prime \prime}}{(n-i) !}=0, n=1,2,3, \ldots
$$

and

$$
\begin{aligned}
& \frac{c_{0}(n, \alpha)}{(n-1) !}+\sum_{i=1}^{n-1} \frac{k_{i}^{\prime \prime}}{(n-i-1) !} \\
& \quad=-\left(\begin{array}{c}
n+\alpha \\
n-2
\end{array}\right) \frac{1}{(n-1) !}+\left(\begin{array}{c}
n+\alpha \\
n-1
\end{array}\right)\left[\frac{b_{0}(n, \alpha)}{(n-1) !}+\sum_{i=1}^{n-1} \frac{k_{i}^{\prime}}{(n-i-1) !}\right], n=2,3,4, \ldots
\end{aligned}
$$

Hence, since $k_{i}^{\prime \prime}$ is independent of $n$, we obtain by (53)

$$
c_{0}(n, \alpha)-c_{0}(n-1, \alpha)=\frac{1}{(\alpha+1)(\alpha+2)}\left(\begin{array}{c}
n+\alpha \\
n-1
\end{array}\right)\left(\begin{array}{c}
n+\alpha+1 \\
n-2
\end{array}\right), n=2,3,4, \ldots
$$

Since $c_{0}(1, \alpha)=b_{0}(1, \alpha)$, this proves that $c_{0}(n, \alpha)=b_{0}(1, \alpha)+\gamma_{0}(n, \alpha), \quad n=$ $1,2,3, \ldots$, where

$$
\gamma_{0}(n, \alpha)=\frac{1}{(\alpha+1)(\alpha+2)} \sum_{k=1}^{n}\left(\begin{array}{c}
k+\alpha \\
k-1
\end{array}\right)\left(\begin{array}{c}
k+\alpha+1 \\
k-2
\end{array}\right), n=1,2,3, \ldots
$$

By using Lemma 4 we see that this equals (17).

Now we will show that every solution of (61) also satisfies (60) and that every solution of (60) also satisfies (59). In order to do this we write (59), (60) and (61) in the form

$$
\sum_{i=0}^{\infty} c_{i}(x) D^{i+k} L_{n}^{(\alpha)}(x)=F_{n}^{(k)}(x), n=0,1,2, \ldots, k=0,1,2,
$$


respectively, where

$$
\begin{aligned}
(-1)^{k+1} F_{n}^{(k)}(x)=\frac{n(\alpha+k)-(\alpha+1)}{(\alpha+1)(\alpha+k+1)}\left(\begin{array}{c}
n+\alpha \\
n-2
\end{array}\right)\left(\begin{array}{c}
n+\alpha \\
n-k
\end{array}\right) D^{2} L_{n+1}^{(\alpha)}(x) \\
+\frac{2}{\alpha+1}\left(\begin{array}{c}
n+\alpha \\
n-2
\end{array}\right)\left(\begin{array}{c}
n+\alpha \\
n-k
\end{array}\right) D^{3} L_{n+1}^{(\alpha)}(x) \\
+\frac{1}{\alpha+1}\left(\begin{array}{c}
n+\alpha \\
n-k-1
\end{array}\right) \sum_{i=0}^{\infty} a_{i}(x) D^{i+2} L_{n}^{(\alpha)}(x) \\
+\left(\begin{array}{c}
n+\alpha \\
n-k
\end{array}\right) \sum_{i=0}^{\infty} b_{i}(x) D^{i+1} L_{n}^{(\alpha)}(x), k=0,1,2 .
\end{aligned}
$$

Suppose that $\left\{c_{i}(x)\right\}_{i=1}^{\infty}$ is a solution of (61). Then we find from

$$
\sum_{i=1}^{\infty} c_{i}(x) D^{i+2} L_{n}^{(\alpha)}(x)=F_{n}^{(2)}(x)-c_{0}(n, \alpha) D^{2} L_{n}^{(\alpha)}(x), n=0,1,2, \ldots,
$$

and (34) that

$$
\begin{aligned}
& \sum_{i=1}^{\infty} c_{i}(x) D^{i+1} L_{n}^{(\alpha)}(x)=\sum_{i=1}^{\infty} c_{i}(x) D^{i+2} L_{n}^{(\alpha)}(x)-\sum_{i=1}^{\infty} c_{i}(x) D^{i+2} L_{n+1}^{(\alpha)}(x) \\
&=F_{n}^{(2)}(x)-c_{0}(n, \alpha) D^{2} L_{n}^{(\alpha)}(x)-F_{n+1}^{(2)}(x)+c_{0}(n+1, \alpha) D^{2} L_{n+1}^{(\alpha)}(x)
\end{aligned}
$$

for $n=0,1,2, \ldots$ Now we use (35) to obtain for $n=0,1,2, \ldots$

$$
\sum_{i=0}^{\infty} c_{i}(x) D^{i+1} L_{n}^{(\alpha)}(x)=F_{n}^{(2)}(x)-F_{n+1}^{(2)}(x)+\left[c_{0}(n+1, \alpha)-c_{0}(n, \alpha)\right] D^{2} L_{n+1}^{(\alpha)}(x) .
$$

So it remains to show that the right-hand side equals $F_{n}^{(1)}(x)$. This can be achieved by straightforward but tedious computations. First we use (49), (35) and (46) to find that

$$
\begin{aligned}
& a_{0}(n+1, \alpha) D^{2} L_{n+1}^{(\alpha)}(x)+\sum_{i=1}^{\infty} a_{i}(x) D^{i+2} L_{n+1}^{(\alpha)}(x) \\
& =a_{0}(n, \alpha)\left[D^{2} L_{n}^{(\alpha)}(x)-D L_{n}^{(\alpha)}(x)\right]+\left(\begin{array}{c}
n+\alpha+1 \\
n
\end{array}\right) D^{2} L_{n+1}^{(\alpha)}(x) \\
& \quad+\sum_{i=1}^{\infty} a_{i}(x)\left[D^{i+2} L_{n}^{(\alpha)}(x)-D^{i+1} L_{n}^{(\alpha)}(x)\right] \\
& =\sum_{i=0}^{\infty} a_{i}(x) D^{i+2} L_{n}^{(\alpha)}(x)-\sum_{i=0}^{\infty} a_{i}(x) D^{i+1} L_{n}^{(\alpha)}(x)+\left(\begin{array}{c}
n+\alpha+1 \\
n
\end{array}\right) D^{2} L_{n+1}^{(\alpha)}(x) \\
& =\sum_{i=0}^{\infty} a_{i}(x) D^{i+2} L_{n}^{(\alpha)}(x)+\left[\left(\begin{array}{c}
n+\alpha \\
n-1
\end{array}\right)+\left(\begin{array}{c}
n+\alpha+1 \\
n
\end{array}\right)\right] D^{2} L_{n+1}^{(\alpha)}(x), n=0,1,2, \ldots .
\end{aligned}
$$


In a similar way we use (56), (35) and (50) to find that

$$
\begin{aligned}
b_{0}(n+ & 1, \alpha) D L_{n+1}^{(\alpha)}(x)+\sum_{i=1}^{\infty} b_{i}(x) D^{i+1} L_{n+1}^{(\alpha)}(x) \\
= & b_{0}(n, \alpha)\left[D L_{n}^{(\alpha)}(x)-L_{n}^{(\alpha)}(x)\right]+\frac{n(\alpha+2)+1}{(\alpha+1)(\alpha+3)}\left(\begin{array}{c}
n+\alpha+1 \\
n-1
\end{array}\right) D L_{n+1}^{(\alpha)}(x) \\
& +\sum_{i=1}^{\infty} b_{i}(x)\left[D^{i+1} L_{n}^{(\alpha)}(x)-D^{i} L_{n}^{(\alpha)}(x)\right] \\
= & \sum_{i=0}^{\infty} b_{i}(x) D^{i+1} L_{n}^{(\alpha)}(x)-\sum_{i=0}^{\infty} b_{i}(x) D^{i} L_{n}^{(\alpha)}(x) \\
& +\frac{n(\alpha+2)+1}{(\alpha+1)(\alpha+3)}\left(\begin{array}{c}
n+\alpha+1 \\
n-1
\end{array}\right) D L_{n+1}^{(\alpha)}(x) \\
= & \sum_{i=0}^{\infty} b_{i}(x) D^{i+1} L_{n}^{(\alpha)}(x)+\frac{n(\alpha+2)+1}{(\alpha+1)(\alpha+3)}\left(\begin{array}{c}
n+\alpha+1 \\
n-1
\end{array}\right) D L_{n+1}^{(\alpha)}(x) \\
& -\frac{1}{\alpha+1}\left(\begin{array}{c}
n+\alpha \\
n-1
\end{array}\right)\left[(n-1) D^{2} L_{n+1}^{(\alpha)}(x)+2 D^{3} L_{n+1}^{(\alpha)}(x)\right], n=1,2,3, \ldots
\end{aligned}
$$

Now we use (35) to obtain

$$
\begin{aligned}
& F_{n}^{(2)}(x)-F_{n+1}^{(2)}(x) \\
& =\frac{1}{\alpha+1}\left(\begin{array}{c}
n+\alpha \\
n-2
\end{array}\right) \sum_{i=0}^{\infty} a_{i}(x) D^{i+2} L_{n}^{(\alpha)}(x)+\left(\begin{array}{c}
n+\alpha \\
n-1
\end{array}\right) \sum_{i=0}^{\infty} b_{i}(x) D^{i+1} L_{n}^{(\alpha)}(x) \\
& +\frac{2}{\alpha+1}\left(\begin{array}{c}
n+\alpha \\
n-2
\end{array}\right)\left(\begin{array}{c}
n+\alpha \\
n-1
\end{array}\right) D^{3} L_{n+1}^{(\alpha)}(x)+\left(\begin{array}{c}
n+\alpha \\
n-2
\end{array}\right) D^{2} L_{n+1}^{(\alpha)}(x) \\
& -\frac{1}{(\alpha+1)(\alpha+2)}\left(\begin{array}{c}
n+\alpha+1 \\
n
\end{array}\right)\left(\begin{array}{c}
n+\alpha+2 \\
n-1
\end{array}\right) D^{2} L_{n+1}^{(\alpha)}(x), n=1,2,3, \ldots
\end{aligned}
$$

Finally we use

$$
\begin{aligned}
& c_{0}(n+1, \alpha)-c_{0}(n, \alpha) \\
& \quad=\frac{1}{(\alpha+1)(\alpha+2)}\left(\begin{array}{c}
n+\alpha+1 \\
n
\end{array}\right)\left(\begin{array}{c}
n+\alpha+2 \\
n-1
\end{array}\right), n=1,2,3, \ldots,
\end{aligned}
$$

which was already obtained before, to conclude that

$$
\begin{gathered}
F_{n}^{(2)}(x)-F_{n+1}^{(2)}(x)+\left[c_{0}(n+1, \alpha)-c_{0}(n, \alpha)\right] D^{2} L_{n+1}^{(\alpha)}(x) \\
=F_{n}^{(1)}(x), n=1,2,3, \ldots
\end{gathered}
$$

For $n=0$ we easily find that

$$
F_{0}^{(2)}(x)-F_{1}^{(2)}(x)+\left[c_{0}(1, \alpha)-c_{0}(0, \alpha)\right] D^{2} L_{1}^{(\alpha)}(x)=0=F_{0}^{(1)}(x) .
$$

Now suppose that $\left\{c_{i}(x)\right\}_{i=1}^{\infty}$ is a solution of (60). Then we have, using (34) for $n=0,1,2, \ldots$,

$$
\begin{aligned}
& \sum_{i=1}^{\infty} c_{i}(x) D^{i} L_{n}^{(\alpha)}(x)=\sum_{i=1}^{\infty} c_{i}(x) D^{i+1} L_{n}^{(\alpha)}(x)-\sum_{i=1}^{\infty} c_{i}(x) D^{i+1} L_{n+1}^{(\alpha)}(x) \\
& =F_{n}^{(1)}(x)-c_{0}(n, \alpha) D L_{n}^{(\alpha)}(x)-F_{n+1}^{(1)}(x)+c_{0}(n+1, \alpha) D L_{n+1}^{(\alpha)}(x) .
\end{aligned}
$$


We use (35) again to obtain for $n=0,1,2, \ldots$

$$
\sum_{i=0}^{\infty} c_{i}(x) D^{i} L_{n}^{(\alpha)}(x)=F_{n}^{(1)}(x)-F_{n+1}^{(1)}(x)+\left[c_{0}(n+1, \alpha)-c_{0}(n, \alpha)\right] D L_{n+1}^{(\alpha)}(x) .
$$

Now we have to show that the right-hand side equals $F_{n}^{(0)}(x)$. This can also be achieved by straightforward but again tedious computations. As before, we obtain by using (35)

$$
\begin{aligned}
& F_{n}^{(1)}(x)-F_{n+1}^{(1)}(x) \\
& =-\frac{1}{\alpha+1}\left(\begin{array}{c}
n+\alpha \\
n-1
\end{array}\right) \sum_{i=0}^{\infty} a_{i}(x) D^{i+2} L_{n}^{(\alpha)}(x)-\left(\begin{array}{c}
n+\alpha \\
n
\end{array}\right) \sum_{i=0}^{\infty} b_{i}(x) D^{i+1} L_{n}^{(\alpha)}(x) \\
& -\frac{2}{\alpha+1}\left(\begin{array}{c}
n+\alpha \\
n-2
\end{array}\right)\left(\begin{array}{c}
n+\alpha \\
n
\end{array}\right) D^{3} L_{n+1}^{(\alpha)}(x) \\
& -\frac{n \alpha-(\alpha+1)}{(\alpha+1)^{2}}\left(\begin{array}{c}
n+\alpha \\
n-2
\end{array}\right)\left(\begin{array}{c}
n+\alpha \\
n
\end{array}\right) D^{2} L_{n+1}^{(\alpha)}(x) \\
& -\frac{1}{(\alpha+1)(\alpha+2)}\left(\begin{array}{c}
n+\alpha+1 \\
n
\end{array}\right)\left(\begin{array}{c}
n+\alpha+2 \\
n-1
\end{array}\right) D L_{n+1}^{(\alpha)}(x), n=1,2,3, \ldots
\end{aligned}
$$

Finally we use (64) again to conclude that

$$
\begin{aligned}
& F_{n}^{(1)}(x)-F_{n+1}^{(1)}(x)+\left[c_{0}(n+1, \alpha)-c_{0}(n, \alpha)\right] D L_{n+1}^{(\alpha)}(x) \\
& =F_{n}^{(0)}(x), n=1,2,3, \ldots
\end{aligned}
$$

For $n=0$ we easily find that

$$
\begin{aligned}
& F_{0}^{(1)}(x)-F_{1}^{(1)}(x)+\left[c_{0}(1, \alpha)-c_{0}(0, \alpha)\right] D L_{1}^{(\alpha)}(x) \\
& =-b_{0}(1, \alpha) D L_{1}^{(\alpha)}(x)+b_{0}(1, \alpha) D L_{1}^{(\alpha)}(x)=0=F_{0}^{(0)}(x) .
\end{aligned}
$$

Now we will solve (63). In order to reduce the number of terms involved we use (51) again to find that for $n=0,1,2, \ldots$

$$
\begin{aligned}
& -\left(\begin{array}{c}
n+\alpha \\
n-2
\end{array}\right) \sum_{i=0}^{\infty} b_{i}(x) D^{i+1} L_{n}^{(\alpha)}(x)=\frac{1}{\alpha+2}\left(\begin{array}{c}
n+\alpha \\
n-1
\end{array}\right) \sum_{i=0}^{\infty} b_{i}(x) D^{i+2} L_{n}^{(\alpha)}(x) \\
& +\frac{n(\alpha+2)-(\alpha+1)}{(\alpha+1)(\alpha+3)}\left(\begin{array}{c}
n+\alpha \\
n-2
\end{array}\right) \\
& \quad \times\left[\left(\begin{array}{c}
n+\alpha \\
n-2
\end{array}\right) D^{2} L_{n+1}^{(\alpha)}(x)+\frac{2}{\alpha+2}\left(\begin{array}{c}
n+\alpha \\
n-1
\end{array}\right) D^{3} L_{n+1}^{(\alpha)}(x)\right] .
\end{aligned}
$$

We use this to write (63) in the form

$$
\begin{aligned}
& \sum_{i=1}^{\infty} c_{i}(x) D^{i+2} L_{n}^{(\alpha)}(x) \\
& =-c_{0}(n, \alpha) D^{2} L_{n}^{(\alpha)}(x)-\frac{2}{(\alpha+1)(\alpha+2)}\left(\begin{array}{c}
n+\alpha \\
n-3
\end{array}\right)\left(\begin{array}{c}
n+\alpha \\
n-1
\end{array}\right) D^{3} L_{n+1}^{(\alpha)}(x) \\
& -\frac{1}{\alpha+1}\left(\begin{array}{c}
n+\alpha \\
n-3
\end{array}\right) \sum_{i=0}^{\infty} a_{i}(x) D^{i+2} L_{n}^{(\alpha)}(x)+\frac{1}{\alpha+2}\left(\begin{array}{c}
n+\alpha \\
n-1
\end{array}\right) \sum_{i=0}^{\infty} b_{i}(x) D^{i+2} L_{n}^{(\alpha)}(x)
\end{aligned}
$$

for $n=0,1,2, \ldots$ We remark that it is necessary for solvability that the right-hand side equals zero for $n=0, n=1$ and $n=2$. For $n=0$ and $n=1$ this is trivial. 
For $n=2$ we use (31), (27), (7), (8), (11) and (17) to see that this right-hand side equals

$$
-c_{0}(2, \alpha) D^{2} L_{2}^{(\alpha)}(x)+b_{0}(2, \alpha) D^{2} L_{2}^{(\alpha)}(x)=-\gamma_{0}(2, \alpha)+\beta_{0}(2, \alpha)=0 .
$$

Now we substitute $c_{0}(n, \alpha)=b_{0}(1, \alpha)+\gamma_{0}(n, \alpha), n=3,4,5, \ldots$, to find by using (7) that

$$
\sum_{i=1}^{\infty} c_{i}(x) D^{i+2} L_{n}^{(\alpha)}(x)=b_{0}(1, \alpha) K_{n}(x)+M_{n}(x), n=3,4,5, \ldots
$$

where

$$
K_{n}(x)=\left[\frac{1}{\alpha+2}\left(\begin{array}{c}
n+\alpha \\
n-1
\end{array}\right)-1\right] D^{2} L_{n}^{(\alpha)}(x)+\frac{1}{\alpha+2}\left(\begin{array}{c}
n+\alpha \\
n-1
\end{array}\right) \sum_{i=1}^{\infty} b_{i}^{*}(x) D^{i+2} L_{n}^{(\alpha)}(x)
$$

and

$$
\begin{aligned}
& M_{n}(x)=-\frac{2}{(\alpha+1)(\alpha+2)}\left(\begin{array}{c}
n+\alpha \\
n-3
\end{array}\right)\left(\begin{array}{c}
n+\alpha \\
n-1
\end{array}\right) D^{3} L_{n+1}^{(\alpha)}(x) \\
& -\left[\gamma_{0}(n, \alpha)+\frac{1}{\alpha+1}\left(\begin{array}{c}
n+\alpha \\
n-3
\end{array}\right) a_{0}(n, \alpha)-\frac{1}{\alpha+2}\left(\begin{array}{c}
n+\alpha \\
n-1
\end{array}\right) \beta_{0}(n, \alpha)\right] D^{2} L_{n}^{(\alpha)}(x) \\
& -\frac{1}{\alpha+1}\left(\begin{array}{c}
n+\alpha \\
n-3
\end{array}\right) \sum_{i=1}^{\infty} a_{i}(x) D^{i+2} L_{n}^{(\alpha)}(x)+\frac{1}{\alpha+2}\left(\begin{array}{c}
n+\alpha \\
n-1
\end{array}\right) \sum_{i=1}^{\infty} \beta_{i}(x) D^{i+2} L_{n}^{(\alpha)}(x) .
\end{aligned}
$$

By using (9) we may write, for $k \in\{0,1,2, \ldots\}$ and $n=0,1,2, \ldots$,

$$
\sum_{i=1}^{\infty} b_{i}^{*}(x) D^{i+k} L_{n}^{(\alpha)}(x)=-D^{k} L_{n}^{(\alpha)}(x)+\sum_{i=0}^{\infty} \sum_{j=0}^{i} \frac{(-1)^{j}}{i !}\left(\begin{array}{l}
i \\
j
\end{array}\right)(\alpha+1)_{i-j} x^{j} D^{i+k} L_{n}^{(\alpha)}(x) .
$$

Changing the order of summation, we find that

$$
\begin{aligned}
& \sum_{i=0}^{\infty} \sum_{j=0}^{i} \frac{(-1)^{j}}{i !}\left(\begin{array}{c}
i \\
j
\end{array}\right)(\alpha+1)_{i-j} x^{j} D^{i+k} L_{n}^{(\alpha)}(x) \\
& =\sum_{j=0}^{\infty} \sum_{i=0}^{\infty} \frac{(-1)^{j}}{(i+j) !}\left(\begin{array}{c}
i+j \\
j
\end{array}\right)(\alpha+1)_{i} x^{j} D^{i+j+k} L_{n}^{(\alpha)}(x) \\
& =\sum_{i=0}^{\infty} \frac{(\alpha+1)_{i}}{i !} \sum_{j=0}^{\infty} \frac{(-1)^{j}}{j !} x^{j} D^{i+j+k} L_{n}^{(\alpha)}(x), k=0,1,2, \ldots
\end{aligned}
$$


Now we use (26) to obtain for $i=0,1,2, \ldots$

$$
\begin{aligned}
\sum_{j=0}^{\infty} \frac{(-1)^{j}}{j !} x^{j} D^{i+j+k} L_{n}^{(\alpha)}(x) \\
\quad=\left(\begin{array}{c}
n+\alpha \\
n
\end{array}\right) \sum_{j=0}^{\infty} \frac{(-1)^{j}}{j !} x^{j} \sum_{m=i+j+k}^{\infty} \frac{(-n)_{m}}{(\alpha+1)_{m}} \frac{x^{m-i-j-k}}{(m-i-j-k) !} \\
\quad=\left(\begin{array}{c}
n+\alpha \\
n
\end{array}\right) \sum_{j=0}^{\infty} \sum_{m=j}^{\infty} \frac{(-1)^{j}}{j !} \frac{(-n)_{m+i+k}}{(\alpha+1)_{m+i+k}} \frac{x^{m}}{(m-j) !} \\
\quad=\left(\begin{array}{c}
n+\alpha \\
n
\end{array}\right) \sum_{m=0}^{\infty} \frac{(-n)_{m+i+k}}{(\alpha+1)_{m+i+k}} \frac{x^{m}}{m !} \sum_{j=0}^{m}(-1)^{j}\left(\begin{array}{c}
m \\
j
\end{array}\right) \\
\quad=\left(\begin{array}{c}
n+\alpha \\
n
\end{array}\right) \frac{(-n)_{i+k}}{(\alpha+1)_{i+k}}, k=0,1,2, \ldots
\end{aligned}
$$

Hence, by using the Vandermonde summation formula (24) we find for $n=1,2,3, \ldots$.

$$
\begin{aligned}
& \sum_{i=0}^{\infty} \sum_{j=0}^{i} \frac{(-1)^{j}}{i !}\left(\begin{array}{c}
i \\
j
\end{array}\right)(\alpha+1)_{i-j} x^{j} D^{i+k} L_{n}^{(\alpha)}(x) \\
& =\left(\begin{array}{c}
n+\alpha \\
n
\end{array}\right) \sum_{i=0}^{\infty} \frac{(\alpha+1)_{i}}{i !} \frac{(-n)_{i+k}}{(\alpha+1)_{i+k}} \\
& =\left(\begin{array}{c}
n+\alpha \\
n
\end{array}\right) \frac{(-n)_{k}}{(\alpha+1)_{k}}{ }_{2} F_{1}\left(\begin{array}{c}
-n+k, \alpha+1 \\
\alpha+k+1
\end{array}\right) \\
& =\left(\begin{array}{c}
n+\alpha \\
n
\end{array}\right) \frac{(-n)_{k}}{(\alpha+1)_{k}} \frac{(k)_{n-k}}{(\alpha+k+1)_{n-k}}=\frac{(-n)_{k}}{n \Gamma(k)}, k \in\{0,1,2, \ldots, n\} .
\end{aligned}
$$

This implies that for $n=1,2,3, \ldots$

$$
\sum_{i=1}^{\infty} b_{i}^{*}(x) D^{i+k} L_{n}^{(\alpha)}(x)=\frac{(-n)_{k}}{n \Gamma(k)}-D^{k} L_{n}^{(\alpha)}(x), k=0,1,2, \ldots
$$

Hence

$$
\sum_{i=1}^{\infty} b_{i}^{*}(x) D^{i+2} L_{n}^{(\alpha)}(x)=n-1-D^{2} L_{n}^{(\alpha)}(x), n=1,2,3, \ldots
$$

which implies that

$$
K_{n}(x)=\left(\begin{array}{c}
n+\alpha \\
n-2
\end{array}\right)-D^{2} L_{n}^{(\alpha)}(x), n=3,4,5, \ldots
$$


By using (5), (11) and (62) we obtain

$$
\begin{aligned}
& \gamma_{0}(n, \alpha)+\frac{1}{\alpha+1}\left(\begin{array}{c}
n+\alpha \\
n-3
\end{array}\right) a_{0}(n, \alpha)-\frac{1}{\alpha+2}\left(\begin{array}{c}
n+\alpha \\
n-1
\end{array}\right) \beta_{0}(n, \alpha) \\
& =\frac{1}{\alpha+1}\left[\left(\begin{array}{c}
n+\alpha \\
n-3
\end{array}\right)\left(\begin{array}{c}
n+\alpha+1 \\
n-1
\end{array}\right)-\frac{n(\alpha+2)-\alpha}{(\alpha+2)(\alpha+4)}\left(\begin{array}{c}
n+\alpha \\
n-1
\end{array}\right)\left(\begin{array}{c}
n+\alpha+1 \\
n-2
\end{array}\right)\right] \\
& \quad+\frac{1}{(\alpha+1)(\alpha+2)} \sum_{k=1}^{n}\left(\begin{array}{c}
k+\alpha \\
k-1
\end{array}\right)\left(\begin{array}{c}
k+\alpha+1 \\
k-2
\end{array}\right) \\
& =\frac{2}{(\alpha+1)(\alpha+2)}\left(\begin{array}{c}
n+\alpha \\
n-1
\end{array}\right)\left(\begin{array}{c}
n+\alpha+1 \\
n-3
\end{array}\right) \\
& \quad+\frac{1}{(\alpha+1)(\alpha+2)} \sum_{k=1}^{n-1}\left(\begin{array}{c}
k+\alpha \\
k-1
\end{array}\right)\left(\begin{array}{c}
k+\alpha+1 \\
k-2
\end{array}\right), n=3,4,5, \ldots
\end{aligned}
$$

Hence, we find by using (31) and (62)

$$
\begin{aligned}
& M_{n}(x)=-\frac{1}{\alpha+1}\left(\begin{array}{c}
n+\alpha \\
n-3
\end{array}\right) \sum_{k=1}^{n-2}(-1)^{k} a_{k}(x) L_{n-k-2}^{(\alpha+k+2)}(x) \\
& +\frac{1}{\alpha+2}\left(\begin{array}{c}
n+\alpha \\
n-1
\end{array}\right) \sum_{k=1}^{n-2}(-1)^{k} \beta_{k}(x) L_{n-k-2}^{(\alpha+k+2)}(x) \\
& \quad-\frac{2}{(\alpha+1)(\alpha+2)}\left(\begin{array}{c}
n+\alpha \\
n-1
\end{array}\right)\left[\left(\begin{array}{c}
n+\alpha \\
n-3
\end{array}\right) D^{3} L_{n+1}^{(\alpha)}(x)+\left(\begin{array}{c}
n+\alpha+1 \\
n-3
\end{array}\right) D^{2} L_{n}^{(\alpha)}(x)\right] \\
& -\gamma_{0}(n-1, \alpha) D^{2} L_{n}^{(\alpha)}(x), n=3,4,5, \ldots .
\end{aligned}
$$

Now we apply the inversion formula of Lemma 5 to (65) to find that

$$
c_{i}(x)=b_{0}(1, \alpha) c_{i}^{*}(x)+\gamma_{i}(x), i=1,2,3, \ldots,
$$

where for $i=1,2,3 \ldots$ we have by using (31)

$$
\begin{aligned}
c_{i}^{*}(x) & =(-1)^{i} \sum_{j=1}^{i} L_{i-j}^{(-\alpha-i-3)}(-x)\left[\left(\begin{array}{c}
j+\alpha+2 \\
j
\end{array}\right)-L_{j}^{(\alpha+2)}(x)\right] \\
& =(-1)^{i}\left[\sum_{j=0}^{i}\left(\begin{array}{c}
j+\alpha+2 \\
j
\end{array}\right) L_{i-j}^{(-\alpha-i-3)}(-x)-\sum_{j=0}^{i} L_{i-j}^{(-\alpha-i-3)}(-x) L_{j}^{(\alpha+2)}(x)\right] .
\end{aligned}
$$

Now we use (33) and (28) to find that for $i=1,2,3, \ldots$

$$
\begin{aligned}
\sum_{j=0}^{i}\left(\begin{array}{c}
j+\alpha+2 \\
j
\end{array}\right) L_{i-j}^{(-\alpha-i-3)}(-x) & =\sum_{j=0}^{i}(-1)^{j}\left(\begin{array}{c}
-\alpha-3 \\
j
\end{array}\right) L_{i-j}^{(-\alpha-i-3)}(-x) \\
& =L_{i}^{(-i)}(-x)=\frac{x^{i}}{i !} .
\end{aligned}
$$

This implies, by using (36), that

$$
c_{i}^{*}(x)=(-1)^{i} \frac{x^{i}}{i !}, i=1,2,3, \ldots,
$$

which proves (10). Further we find that

$$
\gamma_{i}(x):=\gamma_{i}^{(1)}(x)+\gamma_{i}^{(2)}(x)+\gamma_{i}^{(3)}(x)+\gamma_{i}^{(4)}(x)+\gamma_{i}^{(5)}(x), i=1,2,3, \ldots,
$$


where for $i=1,2,3, \ldots$

$$
\begin{gathered}
\gamma_{i}^{(1)}(x)=\frac{(-1)^{i+1}}{\alpha+1} \sum_{k=1}^{i}(-1)^{k} a_{k}(x) \sum_{j=k}^{i}\left(\begin{array}{c}
j+\alpha+2 \\
j-1
\end{array}\right) L_{i-j}^{(-\alpha-i-3)}(-x) L_{j-k}^{(\alpha+k+2)}(x), \\
\gamma_{i}^{(2)}(x)=\frac{(-1)^{i}}{\alpha+2} \sum_{k=1}^{i}(-1)^{k} \beta_{k}(x) \sum_{j=k}^{i}\left(\begin{array}{c}
j+\alpha+2 \\
j+1
\end{array}\right) L_{i-j}^{(-\alpha-i-3)}(-x) L_{j-k}^{(\alpha+k+2)}(x), \\
\gamma_{i}^{(3)}(x)=\frac{2(-1)^{i}}{(\alpha+1)(\alpha+2)} \sum_{j=1}^{i}\left(\begin{array}{c}
j+\alpha+2 \\
j-1
\end{array}\right)\left(\begin{array}{c}
j+\alpha+2 \\
j+1
\end{array}\right) L_{i-j}^{(-\alpha-i-3)}(-x) L_{j}^{(\alpha+3)}(x), \\
\gamma_{i}^{(4)}(x)=\frac{2(-1)^{i+1}}{(\alpha+1)(\alpha+2)} \sum_{j=1}^{i}\left(\begin{array}{c}
j+\alpha+2 \\
j+1
\end{array}\right)\left(\begin{array}{c}
j+\alpha+3 \\
j-1
\end{array}\right) L_{i-j}^{(-\alpha-i-3)}(-x) L_{j}^{(\alpha+2)}(x)
\end{gathered}
$$

and

$$
\gamma_{i}^{(5)}(x)=(-1)^{i+1} \sum_{j=1}^{i} \gamma_{0}(j+1, \alpha) L_{i-j}^{(-\alpha-i-3)}(-x) L_{j}^{(\alpha+2)}(x)
$$

Note that for $i=1,2,3, \ldots$ we have by using (37)

(66)

$$
\gamma_{i}^{(1)}(x)=\frac{(-1)^{i+1}}{\alpha+1} \sum_{k=1}^{i}(-1)^{k}(\alpha+4)_{k-1} a_{k}(x) F_{i-k}(\alpha+k+3, \alpha+k+2 ; k ; x)
$$

and

$$
\gamma_{i}^{(2)}(x)=(-1)^{i} \sum_{k=1}^{i}(-1)^{k}(\alpha+3)_{k} \beta_{k}(x) F_{i-k}(\alpha+k+3, \alpha+k+2 ; k+2 ; x) .
$$

By using (40) we easily obtain (19) and (20).

Further we have, by using (39),

$$
\gamma_{i}^{(3)}(x)=\frac{2(-1)^{i}}{(\alpha+1)(\alpha+3)} H_{i}(\alpha+3, \alpha+3, \alpha+3 ; 0,2 ; x), i=1,2,3, \ldots
$$

and

$$
\gamma_{i}^{(4)}(x)=\frac{2(-1)^{i+1}}{(\alpha+1)(\alpha+4)} H_{i}(\alpha+3, \alpha+4, \alpha+2 ; 0,2 ; x), i=1,2,3, \ldots
$$

Now we apply (44) to obtain for $i=1,2,3, \ldots$.

$$
\gamma_{i}^{(3)}(x)=\frac{2}{(\alpha+1)(\alpha+3)} \sum_{j=0}^{i} \sum_{n=0}^{j}(-1)^{j} \frac{(-j)_{n}(\alpha+3)_{i-j+n}(\alpha+3)_{n}}{j !(i-j) ! n !}
$$

$$
\times{ }_{3} \Phi_{2}\left(\begin{array}{c|c}
-i+j, n+\alpha+3, n+\alpha+4 & 1 \\
n, n+2
\end{array}\right) x^{j}
$$


and

$$
\gamma_{i}^{(4)}(x)=-\frac{2}{(\alpha+1)(\alpha+4)} \sum_{j=0}^{i} \sum_{n=0}^{j}(-1)^{j} \frac{(-j)_{n}(\alpha+3)_{i-j+n}(\alpha+4)_{n}}{j !(i-j) ! n !}
$$

$$
\times{ }_{3} \Phi_{2}\left(\begin{array}{c|c}
-i+j, n+\alpha+4, n+\alpha+3 & 1 \\
n, n+2
\end{array}\right) x^{j}
$$

which lead to (21) and (22) by using (43).

Finally we use (17) to find that for $i=1,2,3, \ldots$

$$
\begin{aligned}
\gamma_{i}^{(5)}(x)=\frac{(-1)^{i+1}}{\alpha+1} \sum_{j=1}^{i}\left(\begin{array}{c}
j+\alpha+2 \\
j-1
\end{array}\right){ }_{3} F_{2}\left(\begin{array}{c}
-j+1,-\alpha-2, \alpha+3 \\
2, \alpha+4
\end{array}\right) \\
\times L_{i-j}^{(-\alpha-i-3)}(-x) L_{j}^{(\alpha+2)}(x) \\
=\frac{(-1)^{i+1}}{(\alpha+1)(\alpha+3)} \sum_{j=1}^{i} \sum_{k=0}^{j-1} \frac{(-j+1)_{k}(-\alpha-2)_{k}(\alpha+3)_{k}}{(2)_{k}(\alpha+4)_{k} k !} \\
\times \frac{(\alpha+3)_{j}}{\Gamma(j)} L_{i-j}^{(-\alpha-i-3)}(-x) L_{j}^{(\alpha+2)}(x) \\
=\frac{(-1)^{i+1}}{(\alpha+1)(\alpha+3)} \sum_{k=0}^{i-1} \sum_{j=k+1}^{i} \frac{(-j+1)_{k}(-\alpha-2)_{k}(\alpha+3)_{k}}{(2)_{k}(\alpha+4)_{k} k !} \\
\times \frac{(\alpha+3)_{j}}{\Gamma(j)} L_{i-j}^{(-\alpha-i-3)}(-x) L_{j}^{(\alpha+2)}(x) .
\end{aligned}
$$

Now we write

$$
\frac{(-j+1)_{k}}{\Gamma(j)}=(-1)^{k} \frac{(j-k)_{k}}{\Gamma(j)}=\frac{(-1)^{k}}{\Gamma(j-k)}, k=0,1,2, \ldots,
$$

to obtain by using (37) and (40)

$$
\begin{aligned}
\gamma_{i}^{(5)}(x)= & \frac{(-1)^{i+1}}{(\alpha+1)(\alpha+3)} \sum_{k=0}^{i-1}(-1)^{k} \frac{(-\alpha-2)_{k}(\alpha+3)_{k}}{(2)_{k}(\alpha+4)_{k} k !} \\
& \times \sum_{j=0}^{i} \frac{(\alpha+3)_{j}}{\Gamma(j-k)} L_{i-j}^{(-\alpha-i-3)}(-x) L_{j}^{(\alpha+2)}(x) \\
= & \frac{(-1)^{i+1}}{(\alpha+1)(\alpha+3)} \sum_{k=0}^{i-1}(-1)^{k} \frac{(-\alpha-2)_{k}(\alpha+3)_{k}}{(2)_{k}(\alpha+4)_{k} k !} F_{i}(\alpha+3, \alpha+2 ;-k ; x) \\
= & \frac{(-1)^{i+1}}{(\alpha+1)(\alpha+3)} \sum_{k=0}^{i-1}(-1)^{k} \frac{(-\alpha-2)_{k}(\alpha+3)_{k}}{(2)_{k}(\alpha+4)_{k} k !} \\
& \times \frac{1}{\Gamma(i-k)} \sum_{j=0}^{i}(-1)^{j}\left(\begin{array}{c}
k+\alpha+3 \\
j
\end{array}\right)\left(\begin{array}{c}
k+\alpha+3 \\
i-j
\end{array}\right)(\alpha+3)_{i-j} x^{j},
\end{aligned}
$$

which proves (23).

Now we will show that for nonnegative integer values of $\alpha$ we have

$$
\gamma_{i}(x)=0 \text { for } i>4 \alpha+10 \text {. }
$$


So we assume that $\alpha \in\{0,1,2, \ldots\}$. First we consider $\gamma_{i}^{(1)}(x)$ given by (66). We use (40) to obtain

$F_{i-k}(\alpha+k+3, \alpha+k+2 ; k ; x)=\frac{1}{\Gamma(i)} \sum_{j=0}^{i-k}(-1)^{j}\left(\begin{array}{c}\alpha+3 \\ j\end{array}\right)\left(\begin{array}{c}\alpha+3 \\ i-k-j\end{array}\right)(\alpha+k+3)_{i-k-j} x^{j}$.

The terms of this sum are equal to zero for $j>\alpha+3$ and for $i-k-j>\alpha+3$. Hence all terms vanish if $i-k>2 \alpha+6$. Since $a_{k}(x)=0$ for $k>2 \alpha+4$ we conclude from (66) in the same way that

$$
\gamma_{i}^{(1)}(x)=0 \text { for } i>2 \alpha+4+2 \alpha+6=4 \alpha+10 .
$$

Now we consider $\gamma_{i}^{(2)}(x)$ given by (67). In the same way we have, by using (40),

$$
\begin{aligned}
& F_{i-k}(\alpha+k+3, \alpha+k+2 ; k+2 ; x) \\
& \quad=\frac{1}{\Gamma(i+2)} \sum_{j=0}^{i-k}(-1)^{j}\left(\begin{array}{c}
\alpha+1 \\
j
\end{array}\right)\left(\begin{array}{c}
\alpha+1 \\
i-k-j
\end{array}\right)(\alpha+k+3)_{i-k-j} x^{j} .
\end{aligned}
$$

The terms of this sum are equal to zero for $j>\alpha+1$ and for $i-k-j>\alpha+1$. Hence all terms vanish if $i-k>2 \alpha+2$. Since $\beta_{k}(x)=0$ for $k>2 \alpha+8$, we conclude from (67) that

$$
\gamma_{i}^{(2)}(x)=0 \text { for } i>2 \alpha+8+2 \alpha+2=4 \alpha+10 .
$$

We need the following lemma:

Lemma 8. For $\ell \in\{0,1,2, \ldots\}$ and $b-a \notin\{1,2,3, \ldots\}$ we have

$$
\begin{aligned}
{ }_{3} \Phi_{2} & \left(\begin{array}{c}
-n, a, c+\ell \\
b, c
\end{array} \mid 1\right) \\
& =\frac{1}{\Gamma(n+b)} \sum_{k=0}^{\ell} \frac{(-n)_{k}(-\ell)_{k}(a)_{k}(b-a-k)_{n}}{(a-b+1)_{k} \Gamma(c+k) k !}, n=0,1,2, \ldots .
\end{aligned}
$$

Proof. Let $\ell \in\{0,1,2, \ldots\}$ and $b-a \notin\{1,2,3, \ldots\}$. We have (see for instance [18], $\S 9.1$, formula $(34))$

$$
\begin{aligned}
{ }_{3} \Phi_{2} & \left(\begin{array}{c}
-n, a, c+\ell \\
b, c
\end{array} \mid 1\right) \\
\quad & =\sum_{k=0}^{n}(-1)^{k} \frac{(-n)_{k}(-\ell)_{k}(a)_{k}}{\Gamma(c+k) k !}{ }_{2} \Phi_{1}\left(\begin{array}{c}
-n+k, a+k \\
b+k
\end{array} \mid 1\right), n=0,1,2, \ldots
\end{aligned}
$$

Hence, we obtain by using the Vandermonde summation formula (42)

$$
\begin{gathered}
{ }_{3} \Phi_{2}\left(\begin{array}{c}
-n, a, c+\ell \\
b, c
\end{array} \mid 1\right)=\frac{1}{\Gamma(n+b)} \sum_{k=0}^{n}(-1)^{k} \frac{(-n)_{k}(-\ell)_{k}(a)_{k}(b-a)_{n-k}}{\Gamma(c+k) k !} \\
=\frac{1}{\Gamma(n+b)} \sum_{k=0}^{\ell} \frac{(-n)_{k}(-\ell)_{k}(a)_{k}(b-a-k)_{n}}{(a-b+1)_{k} \Gamma(c+k) k !}, n=0,1,2, \ldots,
\end{gathered}
$$

which proves $(70)$. 
By using (68) and applying (70) twice we obtain for $i=1,2,3, \ldots$

$$
\begin{aligned}
\gamma_{i}^{(3)}(x)= & \frac{2}{(\alpha+1)(\alpha+3)} \sum_{k=0}^{\alpha+2} \frac{(-\alpha-2)_{k}(\alpha+3)_{k}}{(\alpha+4)_{k} k !} \\
& \times \sum_{j=0}^{i}(-1)^{j} \frac{(-i+j)_{k}(\alpha+3)_{i-j}(-k-\alpha-3)_{i-j}}{j !(i-j) !} \\
& \times{ }_{3} \Phi_{2}\left(\begin{array}{c}
-j, i-j+\alpha+3, k+\alpha+3 \mid 1) x^{j} \\
i-j, k+2
\end{array}\right. \\
= & \frac{2(-1)^{i}}{(\alpha+1)(\alpha+3) \Gamma(i)} \sum_{k=0}^{\alpha+2} \sum_{m=0}^{\alpha+1} \frac{(-\alpha-2)_{k}(\alpha+3)_{k}(-\alpha-1)_{m}(\alpha+3)_{m}}{(\alpha+4)_{k}(\alpha+4)_{m} \Gamma(k+m+2) k ! m !} \\
\times & \sum_{j=0}^{i}(-1)^{j}\left(\begin{array}{c}
m+\alpha+3 \\
j
\end{array}\right)\left(\begin{array}{c}
k+\alpha+3 \\
i-j
\end{array}\right)(-i+j)_{k}(-j)_{m}(m+\alpha+3)_{i-j} x^{j} .
\end{aligned}
$$

Note that the terms of the inner sum are equal to zero for $j>m+\alpha+3$ and for $i-j>k+\alpha+3$. Hence all terms vanish if $i>k+m+2 \alpha+6$. This implies that $\gamma_{i}^{(3)}(x)=0$ if $i>k+m+2 \alpha+6$ for all $k \in\{0,1,2, \ldots, \alpha+2\}$ and $m \in\{0,1,2, \ldots, \alpha+1\}$. Hence

$$
\gamma_{i}^{(3)}(x)=0 \text { for } i>\alpha+2+\alpha+1+2 \alpha+6=4 \alpha+9 .
$$

In a similar way we use (69) and apply (70) twice again to find for $i=1,2,3, \ldots$

$$
\begin{aligned}
\gamma_{i}^{(4)}(x)=- & \frac{2}{(\alpha+1)(\alpha+4)} \sum_{k=0}^{\alpha+1} \frac{(-\alpha-1)_{k}(\alpha+4)_{k}}{(\alpha+5)_{k} k !} \\
& \times \sum_{j=0}^{i}(-1)^{j} \frac{(-i+j)_{k}(\alpha+3)_{i-j}(-k-\alpha-4)_{i-j}}{j !(i-j) !} \\
& \times{ }_{3} \Phi_{2}\left(\begin{array}{c}
-j, i-j+\alpha+3, k+\alpha+4 \\
i-j, k+2
\end{array}\right) x^{j} \\
= & \frac{2(-1)^{i+1}}{(\alpha+1)(\alpha+4) \Gamma(i)} \sum_{k=0}^{\alpha+1} \sum_{m=0}^{\alpha+2} \frac{(-\alpha-1)_{k}(\alpha+4)_{k}(-\alpha-2)_{m}(\alpha+3)_{m}}{(\alpha+5)_{k}(\alpha+4)_{m} \Gamma(k+m+2) k ! m !} \\
\times & \sum_{j=0}^{i}(-1)^{j}\left(\begin{array}{c}
m+\alpha+3 \\
j
\end{array}\right)\left(\begin{array}{c}
k+\alpha+4 \\
i-j
\end{array}\right)(-i+j)_{k}(-j)_{m}(m+\alpha+3)_{i-j} x^{j} .
\end{aligned}
$$

The terms of the inner sum are equal to zero for $j>m+\alpha+3$ and for $i-j>k+\alpha+4$. Hence all terms vanish if $i>k+m+2 \alpha+7$. This implies that

$$
\gamma_{i}^{(4)}(x)=0 \text { for } i>\alpha+1+\alpha+2+2 \alpha+7=4 \alpha+10 .
$$

Further we note that for $i=1,2,3, \ldots$ (23) can now be written as

$$
\gamma_{i}^{(5)}(x)=\frac{(-1)^{i+1}}{(\alpha+1)(\alpha+3)} \sum_{k=0}^{\alpha+2}(-1)^{k} \frac{(-\alpha-2)_{k}(\alpha+3)_{k}}{(2)_{k}(\alpha+4)_{k} k !}
$$

$$
\times \frac{1}{\Gamma(i-k)} \sum_{j=0}^{i}(-1)^{j}\left(\begin{array}{c}
k+\alpha+3 \\
j
\end{array}\right)\left(\begin{array}{c}
k+\alpha+3 \\
i-j
\end{array}\right)(\alpha+3)_{i-j} x^{j}
$$


Now we see that the terms of the inner sum are equal to zero for $j>k+\alpha+3$ and for $i-j>k+\alpha+3$. Hence all terms vanish if $i>2 k+2 \alpha+6$. So we conclude that

$$
\gamma_{i}^{(5)}(x)=0 \text { for } i>2 \alpha+4+2 \alpha+6=4 \alpha+10 .
$$

Finally, it is not difficult to see that $\gamma_{4 \alpha+10}^{(3)}(x)=0$ and that $\gamma_{4 \alpha+10}^{(p)}(x)$ reduces to one single term for $p \in\{1,2,4,5\}$. Moreover, we find that

$$
\gamma_{4 \alpha+10}^{(1)}(x)+\gamma_{4 \alpha+10}^{(2)}(x)=\frac{-2 x^{2 \alpha+5}}{(\alpha+1)(\alpha+2) !(\alpha+4) !}=-\gamma_{4 \alpha+10}^{(4)}(x)
$$

and therefore

$$
\gamma_{4 \alpha+10}(x)=\gamma_{4 \alpha+10}^{(5)}(x)=\frac{x^{2 \alpha+5}}{(\alpha+1)(2 \alpha+5)(\alpha+2) !(\alpha+3) !} .
$$

\section{The sums of The COEFFicients}

Earlier we have discovered (see [10] and [11]) that

$$
\sum_{i=1}^{\infty} a_{i}(x)=-\frac{\sin \pi \alpha}{\pi} \frac{x}{(\alpha+2)(\alpha+3)}{ }_{1} F_{1}\left(\begin{array}{c}
1 \\
\alpha+4
\end{array} \mid-x\right), \alpha>-1
$$

In this section we will give a proof of (74). We will also prove that, for $\alpha>-1$,

$$
\sum_{i=1}^{\infty} \beta_{i}(x)=\frac{\sin \pi \alpha}{\pi} \frac{\alpha+2}{(\alpha+1)(\alpha+3)(\alpha+4)}\left[1-x_{2} F_{2}\left(\begin{array}{c}
1, \alpha+3 \\
\alpha+2, \alpha+5
\end{array} \mid-x\right)\right] .
$$

First we define for complex $x$

$$
K(p, q, r ; s ; \alpha, x):=\sum_{i=0}^{\infty} \sum_{j=0}^{i} \frac{(-1)^{i+j}}{\Gamma(i-s)}\left(\begin{array}{c}
\alpha+p \\
j
\end{array}\right)\left(\begin{array}{c}
\alpha+q \\
i-j
\end{array}\right)(\alpha+r)_{i-j} x^{j},
$$

where $p, q, r$ and $s$ are integers with $p, q, r \geq 0$ and $\alpha>-1$. Then we will prove the following lemma:

Lemma 9. For $\alpha \in\{0,1,2, \ldots\}$ we have

$$
K(p, q, r ; s ; \alpha, x)=\sum_{j=0}^{\infty}\left(\begin{array}{c}
\alpha+p \\
j
\end{array}\right) \frac{(j-\alpha-r-s)_{\alpha+q}}{\Gamma(j+\alpha+q-s)} x^{j}
$$

for all complex $x$ and all integers $p, q, r$ and $s$ with $p, q, r \geq 0$.

For $\alpha>-1$ and $\alpha \notin\{0,1,2, \ldots\}$ the right-hand side of (76) converges absolutely for all complex $x$ if $r+s<q$. In that case we have

$$
K(p, q, r ; s ; \alpha, x)=\sum_{j=0}^{\infty}\left(\begin{array}{c}
\alpha+p \\
j
\end{array}\right) \frac{\Gamma(j+q-r-s)}{\Gamma(j+\alpha+q-s) \Gamma(j-\alpha-r-s)} x^{j} .
$$

Proof. First we assume that $\alpha \in\{0,1,2, \ldots\}$. Then the right-hand side of (76) is a finite sum since $\alpha+p$ and $\alpha+q$ are nonnegative integers. So we may change the 
order of summation to obtain for all complex $x$ and all integers $p, q, r$ and $s$ with $p, q, r \geq 0$

$$
\begin{aligned}
K(p, q, r ; s ; \alpha, x) & =\sum_{i=0}^{\infty} \sum_{j=0}^{i} \frac{(-1)^{i+j}}{\Gamma(i-s)}\left(\begin{array}{c}
\alpha+p \\
j
\end{array}\right)\left(\begin{array}{c}
\alpha+q \\
i-j
\end{array}\right)(\alpha+r)_{i-j} x^{j} \\
& =\sum_{j=0}^{\infty} \sum_{i=0}^{\infty} \frac{(-1)^{i}}{\Gamma(i+j-s)}\left(\begin{array}{c}
\alpha+p \\
j
\end{array}\right)\left(\begin{array}{c}
\alpha+q \\
i
\end{array}\right)(\alpha+r)_{i} x^{j} \\
& =\sum_{j=0}^{\infty}\left(\begin{array}{c}
\alpha+p \\
j
\end{array}\right) x^{j} \sum_{i=0}^{\infty} \frac{(-\alpha-q)_{i}(\alpha+r)_{i}}{\Gamma(i+j-s) i !} \\
& =\sum_{j=0}^{\infty}\left(\begin{array}{c}
\alpha+p \\
j
\end{array}\right){ }_{2} \Phi_{1}\left(\begin{array}{c}
-\alpha-q, \alpha+r \\
j-s
\end{array} \mid 1\right) x^{j} .
\end{aligned}
$$

Now we apply Vandermonde's summation formula (42) to find (77).

Now we assume that $\alpha>-1$ and $\alpha \notin\{0,1,2, \ldots\}$. Then (79) holds for all complex $x$ if $r+s<q$, since

$$
\sum_{j=0}^{\infty} \sum_{i=0}^{\infty}\left(\begin{array}{c}
\alpha+p \\
j
\end{array}\right) \frac{(-\alpha-q)_{i}(\alpha+r)_{i}}{\Gamma(i+j-s) i !} x^{j}
$$

converges absolutely for all complex $x$ if $r+s<q$.

In order to prove this, let $x$ be complex and define

$$
u_{i j}:=\left(\begin{array}{c}
\alpha+p \\
j
\end{array}\right) \frac{\Gamma(i-\alpha-q) \Gamma(i+\alpha+r)}{\Gamma(-\alpha-q) \Gamma(\alpha+r) \Gamma(i+j-s) i !} x^{j}, i, j=0,1,2, \ldots .
$$

Now we will show that

$$
\sum_{j=0}^{\infty} \sum_{i=0}^{\infty}\left|u_{i j}\right|
$$

converges if $r+s<q$.

Since

$$
(-1)^{j}\left(\begin{array}{c}
\alpha+p \\
j
\end{array}\right)=\frac{(-\alpha-p)_{j}}{j !}=\frac{\Gamma(j-\alpha-p)}{\Gamma(-\alpha-p) j !} \sim \frac{j^{-\alpha-p-1}}{\Gamma(-\alpha-p)} \text { for } j \rightarrow \infty
$$

and $\alpha+p+1>p \geq 0$, we have

$$
\lim _{j \rightarrow \infty}\left(\begin{array}{c}
\alpha+p \\
j
\end{array}\right)=0
$$

Hence there exists a positive number $M_{\alpha, p}$, independent of $j$, such that

$$
\left|\left(\begin{array}{c}
\alpha+p \\
j
\end{array}\right)\right| \leq M_{\alpha, p}, j=0,1,2, \ldots .
$$

This implies that

$$
\left|u_{i j}\right| \leq C_{\alpha, p, q, r}\left|\frac{\Gamma(i-\alpha-q) \Gamma(i+\alpha+r)}{\Gamma(i+j-s) i !}\right||x|^{j}, i, j=0,1,2, \ldots,
$$

where

$$
C_{\alpha, p, q, r}:=\frac{M_{\alpha, p}}{|\Gamma(-\alpha-q) \Gamma(\alpha+r)|}>0 .
$$

We distinguish two cases: $s<0$ and $s \geq 0$. 
If $s<0$ we have $\Gamma(i+j-s)=(i+j-s-1) ! \geq j !(i-s-1)$ !. So we obtain

$$
\left|u_{i j}\right| \leq C_{\alpha, p, q, r}\left|\frac{\Gamma(i-\alpha-q) \Gamma(i+\alpha+r)}{\Gamma(i-s) \Gamma(i+1)}\right| \frac{|x|^{j}}{j !}, i, j=0,1,2, \ldots .
$$

Since

$$
\left|\frac{\Gamma(i-\alpha-q) \Gamma(i+\alpha+r)}{\Gamma(i-s) \Gamma(i+1)}\right| \sim i^{r+s-q-1} \text { for } i \rightarrow \infty,
$$

we conclude that

$$
\sum_{j=0}^{\infty} \sum_{i=0}^{\infty}\left|u_{i j}\right|<\infty
$$

for $s<0$ if $r+s<q$.

If $s \geq 0$ we find by using (80) that $\left|u_{i j}\right|=0$ if $i+j \leq s$. So we may write

$$
\sum_{j=0}^{\infty} \sum_{i=0}^{\infty}\left|u_{i j}\right|=\sum_{j=0}^{s} \sum_{i=s+1-j}^{\infty}\left|u_{i j}\right|+\sum_{j=s+1}^{\infty} \sum_{i=0}^{\infty}\left|u_{i j}\right| .
$$

For $0 \leq j \leq s$ we have

$$
\left|\frac{\Gamma(i-\alpha-q) \Gamma(i+\alpha+r)}{\Gamma(i+j-s) i !}\right| \sim i^{r+s-q-1-j} \quad \text { for } i \rightarrow \infty
$$

and $r+s-q-1-j \leq r+s-q-1$. Hence

$$
\sum_{j=0}^{s} \sum_{i=s+1-j}^{\infty}\left|u_{i j}\right|<\infty
$$

if $r+s<q$.

For $j \geq s+1$ we may write $\Gamma(i+j-s)=(i+j-s-1) ! \geq i !(j-s-1)$ !. Hence

$$
\left|u_{i j}\right| \leq C_{\alpha, p, q, r}\left|\frac{\Gamma(i-\alpha-q) \Gamma(i+\alpha+r)}{i ! i !}\right| \frac{|x|^{j}}{(j-s-1) !}
$$

for $i=0,1,2, \ldots$ and $j=s+1, s+2, s+3, \ldots$ Since

$$
\left|\frac{\Gamma(i-\alpha-q) \Gamma(i+\alpha+r)}{i ! i !}\right| \sim i^{r-q-2} \text { for } i \rightarrow \infty
$$

and $r-q-2=r+s-q-1-s-1<r+s-q-1$ we conclude that we also have

$$
\sum_{j=s+1}^{\infty} \sum_{i=0}^{\infty}\left|u_{i j}\right|<\infty
$$

if $r+s<q$.

This implies that

$$
\sum_{j=0}^{\infty} \sum_{i=0}^{\infty}\left|u_{i j}\right|<\infty
$$

for $s \geq 0$ if $r+s<q$.

This proves that if $\alpha>-1$ and $\alpha \notin\{0,1,2, \ldots\}$, then

$$
\sum_{j=0}^{\infty} \sum_{i=0}^{\infty}\left|u_{i j}\right|
$$


converges if $r+s<q$. So (79) holds for all complex $x$ if $r+s<q$. Now we apply Gauss' summation formula

$$
{ }_{2} \Phi_{1}\left(\begin{array}{c|c}
a, b & 1 \\
c & 1
\end{array}\right)=\frac{\Gamma(c-a-b)}{\Gamma(c-a) \Gamma(c-b)}, c-a-b>0,
$$

to find (78). This proves Lemma 9.

Note that (78) also holds for $\alpha \in\{0,1,2, \ldots\}$ if $r+s<q$. So for $p=r+s \geq 0$ and $q=r+s+1$ we use (78) and the well-known formula

$$
\frac{1}{\Gamma(z) \Gamma(1-z)}=\frac{\sin \pi z}{\pi}
$$

to find that for all $\alpha>-1$

$$
K(r+s, r+s+1, r ; s ; \alpha, x)=\sum_{j=0}^{\infty} \frac{\sin \pi(j-\alpha-r-s)}{\pi} \frac{\Gamma(\alpha+r+s+1)}{\Gamma(j+\alpha+r+1)} x^{j} .
$$

Now we use

$$
\sin \pi(j-\alpha-r-s)=(-1)^{j+r+s+1} \sin \pi \alpha, j=0,1,2, \ldots,
$$

to obtain for all $\alpha>-1$

$$
K(r+s, r+s+1, r ; s ; \alpha, x)=(-1)^{r+s+1} \frac{\sin \pi \alpha}{\pi} \sum_{j=0}^{\infty} \frac{\Gamma(\alpha+r+s+1)}{\Gamma(j+\alpha+r+1)}(-x)^{j}
$$

for all integers $r$ and $s$ with $r \geq 0$ and $r+s \geq 0$.

Now we find from (6) that for all $\alpha>-1$, by using (76) and (82),

$$
\begin{aligned}
\sum_{i=1}^{\infty} a_{i}(x) & =-x K(1,2,3 ;-2 ; \alpha, x)=-x \frac{\sin \pi \alpha}{\pi} \sum_{j=0}^{\infty} \frac{\Gamma(\alpha+2)}{\Gamma(j+\alpha+4)}(-x)^{j} \\
& =-\frac{\sin \pi \alpha}{\pi} \frac{x}{(\alpha+2)(\alpha+3)}{ }_{1} F_{1}\left(\begin{array}{c}
1 \\
\alpha+4
\end{array} \mid-x\right) .
\end{aligned}
$$

This proves (74).

In a similar way we obtain from (13) and (14) for all $\alpha>-1$, by using (76) and (82),

$$
\begin{aligned}
\sum_{i=2}^{\infty} \beta_{i}^{(1)}(x) & =-\frac{1}{\alpha+1} K(2,3,2 ; 0 ; \alpha, x)=\frac{1}{\alpha+1} \frac{\sin \pi \alpha}{\pi} \sum_{j=0}^{\infty} \frac{\Gamma(\alpha+3)}{\Gamma(j+\alpha+3)}(-x)^{j} \\
& =\frac{\sin \pi \alpha}{\pi} \frac{1}{\alpha+1}{ }_{1} F_{1}\left(\begin{array}{c}
1 \\
\alpha+3
\end{array} \mid-x\right)
\end{aligned}
$$

and

$$
\begin{aligned}
\sum_{i=2}^{\infty} \beta_{i}^{(2)}(x) & =-\frac{2(\alpha+2)}{\alpha+1} K(1,2,3 ;-2 ; \alpha, x) \\
& =-\frac{2(\alpha+2)}{\alpha+1} \frac{\sin \pi \alpha}{\pi} \sum_{j=0}^{\infty} \frac{\Gamma(\alpha+2)}{\Gamma(j+\alpha+4)}(-x)^{j} \\
& =-\frac{\sin \pi \alpha}{\pi} \frac{2}{(\alpha+1)(\alpha+3)}{ }_{1} F_{1}\left(\begin{array}{c}
1 \\
\alpha+4
\end{array} \mid-x\right) .
\end{aligned}
$$


If $p=q=r+s+1$, we also obtain from (78) for all $\alpha>-1$

$$
K(r+s+1, r+s+1, r ; s ; \alpha, x)=\sum_{j=0}^{\infty} \frac{(-\alpha-r-s-1)_{j}}{\Gamma(j+\alpha+r+1) \Gamma(j-\alpha-r-s)}(-x)^{j}
$$

for all integers $r$ and $s$ with $r \geq 0$ and $r+s+1 \geq 0$.

Now we obtain from (15) and (16), by using (76), (83) and (81), for all $\alpha>-1$

$$
\begin{aligned}
\sum_{i=2}^{\infty} \beta_{i}^{(3)}(x)= & -\frac{1}{(\alpha+1)(\alpha+2)(\alpha+3)} K(3,3,1 ; 1 ; \alpha, x) \\
& +\frac{x}{(\alpha+1)(\alpha+2)} K(2,2,2 ;-1 ; \alpha, x) \\
= & -\frac{1}{(\alpha+1)(\alpha+2)(\alpha+3)} \sum_{j=0}^{\infty} \frac{(-\alpha-3)_{j}}{\Gamma(j+\alpha+2) \Gamma(j-\alpha-2)}(-x)^{j} \\
& \quad+\frac{x}{(\alpha+1)(\alpha+2)} \sum_{j=0}^{\infty} \frac{(-\alpha-2)_{j}}{\Gamma(j+\alpha+3) \Gamma(j-\alpha-1)}(-x)^{j} \\
= & -\frac{1}{(\alpha+1)(\alpha+2)(\alpha+3)} \sum_{j=0}^{\infty} \frac{(-\alpha-3)_{j}}{\Gamma(j+\alpha+2) \Gamma(j-\alpha-2)}(-x)^{j} \\
=-\frac{1}{(\alpha+1)(\alpha+2)(\alpha+3)} \frac{(-\alpha-3)_{j}}{\Gamma(\alpha+2) \Gamma(-\alpha-2)}=\frac{\sin \pi \alpha}{\pi} \frac{1}{(\alpha+1)(\alpha+3)} &
\end{aligned}
$$

and

$$
\begin{aligned}
\sum_{i=2}^{\infty} \beta_{i}^{(4)}(x)= & -\frac{1}{(\alpha+1)(\alpha+3)(\alpha+4)} K(4,4,1 ; 2 ; \alpha, x) \\
& +\frac{x}{(\alpha+1)(\alpha+3)} K(3,3,2 ; 0 ; \alpha, x) \\
= & -\frac{1}{(\alpha+1)(\alpha+3)(\alpha+4)} \sum_{j=0}^{\infty} \frac{(-\alpha-4)_{j}}{\Gamma(j+\alpha+2) \Gamma(j-\alpha-3)}(-x)^{j} \\
& \quad+\frac{x}{(\alpha+1)(\alpha+3)} \sum_{j=0}^{\infty} \frac{(-\alpha-3)_{j}}{\Gamma(j+\alpha+3) \Gamma(j-\alpha-2)}(-x)^{j} \\
= & -\frac{1}{(\alpha+1)(\alpha+3)(\alpha+4)} \sum_{j=0}^{\infty} \frac{(-\alpha-4)_{j}}{\Gamma(j+\alpha+2) \Gamma(j-\alpha-3)}(-x)^{j} \\
=-\frac{1}{(\alpha+1)(\alpha+3)(\alpha+4)} \sum_{j=1}^{\infty} \frac{(-\alpha-4)_{j}}{\Gamma(j+\alpha+2) \Gamma(j-\alpha-3)}(-x)^{j} & \frac{1}{(\alpha+1)(\alpha+3)(\alpha+4)} \frac{\sin \pi \alpha}{\Gamma(\alpha+2) \Gamma(-\alpha-3)} \frac{\alpha+2}{(\alpha+1)(\alpha+4)}
\end{aligned}
$$


Hence, since $\beta_{1}(x)=0$, we have

$$
\begin{aligned}
& \sum_{i=1}^{\infty} \beta_{i}(x)=\sum_{i=2}^{\infty}\left[\beta_{i}^{(1)}(x)+\beta_{i}^{(2)}(x)+\beta_{i}^{(3)}(x)+\beta_{i}^{(4)}(x)\right] \\
& =\frac{\sin \pi \alpha}{\pi} \frac{1}{\alpha+1}\left[{ }_{1} F_{1}\left(\begin{array}{c}
1 \\
\alpha+3
\end{array} \mid-x\right)-\frac{2}{\alpha+3}{ }_{1} F_{1}\left(\begin{array}{c}
1 \\
\alpha+4
\end{array} \mid-x\right)-\frac{\alpha^{2}+4 \alpha+2}{(\alpha+3)(\alpha+4)}\right] \\
& =\frac{\sin \pi \alpha}{\pi} \frac{1}{(\alpha+1)(\alpha+3)}\left[\frac{\alpha+2}{\alpha+4}+\sum_{k=1}^{\infty} \frac{\alpha+k+1}{(\alpha+4)_{k}}(-x)^{k}\right] \\
& =\frac{\sin \pi \alpha}{\pi} \frac{\alpha+2}{(\alpha+1)(\alpha+3)(\alpha+4)}\left[1-x_{2} F_{2}\left(\begin{array}{c}
1, \alpha+3 \\
\alpha+2, \alpha+5
\end{array} \mid-x\right)\right], \alpha>-1,
\end{aligned}
$$

which proves (75).

Note that for nonnegative integer values of $\alpha$ this implies that

$$
\sum_{i=1}^{2 \alpha+4} a_{i}(x)=0 \text { and } \sum_{i=1}^{2 \alpha+8} \beta_{i}(x)=0
$$

Finally we will show that for nonnegative integer values of $\alpha$ we also have

$$
\sum_{i=1}^{4 \alpha+10} \gamma_{i}(x)=0
$$

Therefore we assume that $\alpha \in\{0,1,2, \ldots\}$. Now we will use (77). Note that if $p=q=r+s \geq 0$ we obtain

$$
\begin{aligned}
K(r+s, r+s, r ; s ; \alpha, x) & =\sum_{j=0}^{\infty} \frac{(-\alpha-r-s)_{j+\alpha+r+s}}{\Gamma(j+\alpha+r) j !}(-x)^{j}=\frac{(-\alpha-r-s)_{\alpha+r+s}}{\Gamma(\alpha+r)} \\
& =(-1)^{\alpha+r+s} \frac{\Gamma(\alpha+r+s+1)}{\Gamma(\alpha+r)}=(-1)^{\alpha+r+s} \frac{(\alpha+1)_{r+s}}{(\alpha+1)_{r-1}}
\end{aligned}
$$

for all integers $r$ and $s$ with $r \geq 1$ and $r+s \geq 0$.

From (66) we easily obtain, by changing the order of summation,

$$
\sum_{i=1}^{\infty} \gamma_{i}^{(1)}(x)=\frac{1}{\alpha+1} \sum_{k=1}^{\infty}(\alpha+4)_{k-1} a_{k}(x) \sum_{i=0}^{\infty}(-1)^{i+1} F_{i}(\alpha+k+3, \alpha+k+2 ; k ; x) .
$$

Note that all summations are in fact finite. Now we use (40), (76) and (84) to obtain

$$
\begin{aligned}
& \sum_{i=0}^{\infty}(-1)^{i} F_{i}(\alpha+k+3, \alpha+k+2 ; k ; x) \\
& =\sum_{i=0}^{\infty} \sum_{j=0}^{i} \frac{(-1)^{i+j}}{\Gamma(k+i)}\left(\begin{array}{c}
\alpha+3 \\
j
\end{array}\right)\left(\begin{array}{c}
\alpha+3 \\
i-j
\end{array}\right)(\alpha+k+3)_{i-j} x^{j} \\
& =K(3,3, k+3 ;-k ; \alpha, x)=\frac{(-1)^{\alpha+1}}{(\alpha+4)_{k-1}}, k=1,2,3, \ldots
\end{aligned}
$$

Hence

$$
\sum_{i=1}^{\infty} \gamma_{i}^{(1)}(x)=\frac{(-1)^{\alpha}}{\alpha+1} \sum_{k=1}^{\infty} a_{k}(x)=0
$$


In the same way we find from (67), by changing the order of summation, that

$$
\sum_{i=1}^{\infty} \gamma_{i}^{(2)}(x)=\sum_{k=1}^{\infty}(\alpha+3)_{k} \beta_{k}(x) \sum_{i=0}^{\infty}(-1)^{i} F_{i}(\alpha+k+3, \alpha+k+2 ; k+2 ; x) .
$$

Again we use (40), (76) and (84) to find that

$$
\begin{aligned}
& \sum_{i=0}^{\infty}(-1)^{i} F_{i}(\alpha+k+3, \alpha+k+2 ; k+2 ; x) \\
& =\sum_{i=0}^{\infty} \sum_{j=0}^{i} \frac{(-1)^{i+j}}{\Gamma(k+i+2)}\left(\begin{array}{c}
\alpha+1 \\
j
\end{array}\right)\left(\begin{array}{c}
\alpha+1 \\
i-j
\end{array}\right)(\alpha+k+3)_{i-j} x^{j} \\
& =K(1,1, k+3 ;-k-2 ; \alpha, x)=\frac{(-1)^{\alpha+1}}{(\alpha+2)_{k+1}}, k=1,2,3, \ldots
\end{aligned}
$$

Hence

$$
\sum_{i=1}^{\infty} \gamma_{i}^{(2)}(x)=\frac{(-1)^{\alpha+1}}{\alpha+2} \sum_{k=1}^{\infty} \beta_{k}(x)=0 .
$$

By using (71) we obtain

$$
\begin{aligned}
& \sum_{i=1}^{\infty} \gamma_{i}^{(3)}(x) \\
& =\frac{2}{(\alpha+1)(\alpha+3)} \sum_{k=0}^{\alpha+2} \sum_{m=0}^{\alpha+1} \frac{(-\alpha-2)_{k}(\alpha+3)_{k}(-\alpha-1)_{m}(\alpha+3)_{m}}{(\alpha+4)_{k}(\alpha+4)_{m} \Gamma(k+m+2) k ! m !} \\
& \quad \times \sum_{i=0}^{\infty} \sum_{j=0}^{i} \frac{(-1)^{i+j}}{\Gamma(i)}\left(\begin{array}{c}
m+\alpha+3 \\
j
\end{array}\right)\left(\begin{array}{c}
k+\alpha+3 \\
i-j
\end{array}\right)(-i+j)_{k}(-j)_{m}(m+\alpha+3)_{i-j} x^{j} .
\end{aligned}
$$

Changing the order of summation, we find that

$$
\begin{aligned}
& \sum_{i=0}^{\infty} \sum_{j=0}^{i} \frac{(-1)^{i+j}}{\Gamma(i)}\left(\begin{array}{c}
m+\alpha+3 \\
j
\end{array}\right)\left(\begin{array}{c}
k+\alpha+3 \\
i-j
\end{array}\right)(-i+j)_{k}(-j)_{m}(m+\alpha+3)_{i-j} x^{j} \\
& =\sum_{j=0}^{\infty}\left(\begin{array}{c}
m+\alpha+3 \\
j
\end{array}\right)(-j)_{m} x^{j} \sum_{i=0}^{\infty} \frac{(-1)^{i}}{\Gamma(i+j)}\left(\begin{array}{c}
k+\alpha+3 \\
i
\end{array}\right)(-i)_{k}(m+\alpha+3)_{i} .
\end{aligned}
$$

Since $(-i)_{k}=0$ for $i<k$, we have, by using the Vandermonde summation formula $(42)$,

$$
\begin{aligned}
S(j, k, m ; \alpha) & :=\sum_{i=0}^{\infty} \frac{(-1)^{i}}{\Gamma(i+j)}\left(\begin{array}{c}
k+\alpha+3 \\
i
\end{array}\right)(-i)_{k}(m+\alpha+3)_{i} \\
& =\sum_{i=k}^{\infty} \frac{(-k-\alpha-3)_{i}(-i)_{k}(m+\alpha+3)_{i}}{\Gamma(i+j) i !} \\
& =(-1)^{k}(-k-\alpha-3)_{k}(m+\alpha+3)_{k 2} \Phi_{1}\left(\begin{array}{c}
-\alpha-3, k+m+\alpha+3 \\
k+j
\end{array} \mid 1\right) \\
(85) \quad & =(\alpha+4)_{k}(m+\alpha+3)_{k} \frac{(j-m-\alpha-3)_{\alpha+3}}{\Gamma(k+j+\alpha+3)}, k=0,1,2, \ldots
\end{aligned}
$$


for every arbitrary $j$ and $m$ and for each $\alpha \in\{0,1,2, \ldots\}$. Hence

$$
\begin{aligned}
& \sum_{i=0}^{\infty} \sum_{j=0}^{i} \frac{(-1)^{i+j}}{\Gamma(i)}\left(\begin{array}{c}
m+\alpha+3 \\
j
\end{array}\right)\left(\begin{array}{c}
k+\alpha+3 \\
i-j
\end{array}\right)(-i+j)_{k}(-j)_{m}(m+\alpha+3)_{i-j} x^{j} \\
& =(\alpha+4)_{k}(m+\alpha+3)_{k} \sum_{j=0}^{\infty}\left(\begin{array}{c}
m+\alpha+3 \\
j
\end{array}\right) \frac{(-j)_{m}(j-m-\alpha-3)_{\alpha+3}}{\Gamma(k+j+\alpha+3)} x^{j} \\
& =(\alpha+4)_{k}(m+\alpha+3)_{k} \sum_{j=0}^{\infty}(-1)^{j} \frac{(-j)_{m}(-m-\alpha-3)_{j+\alpha+3}}{\Gamma(k+j+\alpha+3) j !} x^{j} \\
& =(\alpha+4)_{k}(m+\alpha+3)_{k}(-1)^{m} \frac{(-m)_{m}(-m-\alpha-3)_{m+\alpha+3}}{\Gamma(k+m+\alpha+3) m !} x^{m} \\
& =(\alpha+4)_{k}(m+\alpha+3)(-1)^{m+\alpha+1} x^{m}, k, m=0,1,2, \ldots,
\end{aligned}
$$

since $(-j)_{m}(-m-\alpha-3)_{j+\alpha+3}=0$ for all $j$ except $j=m$.

This implies that

$$
\begin{aligned}
\sum_{i=1}^{\infty} \gamma_{i}^{(3)}(x)= & \frac{2(-1)^{\alpha+1}}{(\alpha+1)(\alpha+3)} \sum_{k=0}^{\alpha+2} \sum_{m=0}^{\alpha+1}(-1)^{m} \\
& \quad \times \frac{(-\alpha-2)_{k}(\alpha+3)_{k}(-\alpha-1)_{m}(\alpha+3)_{m}}{(\alpha+4)_{m} \Gamma(k+m+2) k ! m !}(m+\alpha+3) x^{m} \\
= & \frac{2(-1)^{\alpha+1}}{\alpha+1} \sum_{m=0}^{\alpha+1}(-1)^{m} \frac{(-\alpha-1)_{m}}{m !} x^{m} \sum_{k=0}^{\alpha+2} \frac{(-\alpha-2)_{k}(\alpha+3)_{k}}{\Gamma(k+m+2) k !} .
\end{aligned}
$$

Finally, we use the Vandermonde summation formula (42) again to obtain

$$
\sum_{k=0}^{\alpha+2} \frac{(-\alpha-2)_{k}(\alpha+3)_{k}}{\Gamma(k+m+2) k !}={ }_{2} \Phi_{1}\left(\begin{array}{c}
-\alpha-2, \alpha+3 \\
m+2
\end{array} \mid 1\right)=\frac{(m-\alpha-1)_{\alpha+2}}{\Gamma(m+\alpha+4)}
$$

Hence

$$
\sum_{i=1}^{\infty} \gamma_{i}^{(3)}(x)=\frac{2(-1)^{\alpha+1}}{\alpha+1} \sum_{m=0}^{\alpha+1} \frac{(-\alpha-1)_{m+\alpha+2}}{\Gamma(m+\alpha+4) m !}(-x)^{m}=0 .
$$

Furthermore, by using (72) we obtain

$$
\begin{aligned}
& \sum_{i=1}^{\infty} \gamma_{i}^{(4)}(x) \\
& =\frac{2}{(\alpha+1)(\alpha+4)} \sum_{k=0}^{\alpha+1} \sum_{m=0}^{\alpha+2} \frac{(-\alpha-1)_{k}(\alpha+4)_{k}(-\alpha-2)_{m}(\alpha+3)_{m}}{(\alpha+5)_{k}(\alpha+4)_{m} \Gamma(k+m+2) k ! m !} \\
& \quad \times \sum_{i=0}^{\infty} \sum_{j=0}^{i} \frac{(-1)^{i+j+1}}{\Gamma(i)}\left(\begin{array}{c}
m+\alpha+3 \\
j
\end{array}\right)\left(\begin{array}{c}
k+\alpha+4 \\
i-j
\end{array}\right)(-i+j)_{k}(-j)_{m}(m+\alpha+3)_{i-j} x^{j} .
\end{aligned}
$$


Changing the order of summation, we find by using (85) that

$$
\begin{aligned}
& \sum_{i=0}^{\infty} \sum_{j=0}^{i} \frac{(-1)^{i+j+1}}{\Gamma(i)}\left(\begin{array}{c}
m+\alpha+3 \\
j
\end{array}\right)\left(\begin{array}{c}
k+\alpha+4 \\
i-j
\end{array}\right)(-i+j)_{k}(-j)_{m}(m+\alpha+3)_{i-j} x^{j} \\
& =-\sum_{j=0}^{\infty}\left(\begin{array}{c}
m+\alpha+3 \\
j
\end{array}\right)(-j)_{m} x^{j} \sum_{i=0}^{\infty} \frac{(-1)^{i}}{\Gamma(i+j)}\left(\begin{array}{c}
k+\alpha+4 \\
i
\end{array}\right)(-i)_{k}(m+\alpha+3)_{i} \\
& =-\sum_{j=0}^{\infty}\left(\begin{array}{c}
m+\alpha+3 \\
j
\end{array}\right)(-j)_{m} x^{j} S(j, k, m-1 ; \alpha+1) \\
& =-(\alpha+5)_{k}(m+\alpha+3)_{k} \sum_{j=0}^{\infty}\left(\begin{array}{c}
m+\alpha+3 \\
j
\end{array}\right) \frac{(-j)_{m}(j-m-\alpha-3)_{\alpha+4}}{\Gamma(k+j+\alpha+4)} x^{j} \\
& =-(\alpha+5)_{k}(m+\alpha+3)_{k} \sum_{j=0}^{\infty}(-1)^{j} \frac{(-j)_{m}(-m-\alpha-3)_{j+\alpha+4}}{\Gamma(k+j+\alpha+4) j !} x^{j}, \\
& k, m=0,1,2, \ldots
\end{aligned}
$$

Since $(-j)_{m}(-m-\alpha-3)_{j+\alpha+4}=0$ for all $j$, this immediately implies that

$$
\sum_{i=1}^{\infty} \gamma_{i}^{(4)}(x)=0
$$

Finally, we have, as before, by using (73),

$$
\begin{aligned}
\sum_{i=1}^{\infty} \gamma_{i}^{(5)}(x)=- & \frac{1}{(\alpha+1)(\alpha+3)} \sum_{k=0}^{\alpha+2}(-1)^{k} \frac{(-\alpha-2)_{k}(\alpha+3)_{k}}{(2)_{k}(\alpha+4)_{k} k !} \\
& \times \sum_{i=0}^{\infty} \sum_{j=0}^{i} \frac{(-1)^{i+j}}{\Gamma(i-k)}\left(\begin{array}{c}
k+\alpha+3 \\
j
\end{array}\right)\left(\begin{array}{c}
k+\alpha+3 \\
i-j
\end{array}\right)(\alpha+3)_{i-j} x^{j} .
\end{aligned}
$$

By using (76) and (84) using we find that

$$
\begin{aligned}
& \sum_{i=0}^{\infty} \sum_{j=0}^{i} \frac{(-1)^{i+j}}{\Gamma(i-k)}\left(\begin{array}{c}
k+\alpha+3 \\
j
\end{array}\right)\left(\begin{array}{c}
k+\alpha+3 \\
i-j
\end{array}\right)(\alpha+3)_{i-j} x^{j} \\
& =K(k+3, k+3,3 ; k ; \alpha, x)=(-1)^{\alpha+k+1}(\alpha+3)_{k+1}, k=0,1,2, \ldots
\end{aligned}
$$

Hence, using (42) again,

$$
\sum_{i=1}^{\infty} \gamma_{i}^{(5)}(x)=\frac{(-1)^{\alpha}}{\alpha+1}{ }_{2} \Phi_{1}\left(\begin{array}{c|c}
-\alpha-2, \alpha+3 \\
2
\end{array} \mid 1\right)=\frac{(-1)^{\alpha}}{\alpha+1} \frac{(-\alpha-1)_{\alpha+2}}{\Gamma(\alpha+4)}=0
$$

From (18) and the fact that $\gamma_{i}(x)=0$ for $i>4 \alpha+10$ we conclude that

$$
\sum_{i=1}^{\infty} \gamma_{i}(x)=\sum_{i=1}^{4 \alpha+10} \gamma_{i}(x)=0
$$

for all $\alpha \in\{0,1,2, \ldots\}$. 


\section{REMARKS}

In this section we list some facts we encountered during the research. First of all we remark that

$$
L_{n}^{(-n)}(-x)=\frac{x^{n}}{n !}=\sum_{k=0}^{n}(-1)^{k}\left(\begin{array}{c}
n+\alpha \\
n-k
\end{array}\right) L_{k}^{(\alpha)}(x), n=0,1,2, \ldots
$$

This formula can also be used for inversion instead of Lemma 5 in the following way (compare with Lemma 5): Suppose that for $m \in\{0,1,2, \ldots\}$ we have the system of equations

$$
\sum_{i=1}^{\infty} A_{i}(x) D^{i+m} L_{n}^{(\alpha)}(x)=F_{n}(x), n=m+1, m+2, m+3, \ldots,
$$

where $\left\{A_{i}(x)\right\}_{i=1}^{\infty}$ are independent of $n$. Then we simply find that

$$
\sum_{i=1}^{\infty} A_{i}(x) D^{i+m} \frac{x^{n}}{n !}=\sum_{k=m+1}^{n}(-1)^{k}\left(\begin{array}{c}
n+\alpha \\
n-k
\end{array}\right) F_{k}(x), n=m+1, m+2, m+3, \ldots
$$

Since the generating function

$$
e^{x t}=\sum_{n=0}^{\infty} \frac{x^{n}}{n !} t^{n}
$$

has the trivial inverse $e^{-x t}$, we now conclude that

$$
A_{i}(x)=(-1)^{m} \sum_{j=1}^{i} \frac{(-x)^{i-j}}{(i-j) !} \sum_{k=1}^{j}(-1)^{k}\left(\begin{array}{c}
m+j+\alpha \\
j-k
\end{array}\right) F_{m+k}(x), i=1,2,3, \ldots
$$

However, for our purposes this method turned out to be very inconvenient.

Further, we have from (2)

$$
\begin{aligned}
& A_{0}=1+M\left(\begin{array}{c}
n+\alpha \\
n-1
\end{array}\right)+\frac{n(\alpha+2)-(\alpha+1)}{(\alpha+1)(\alpha+3)} N\left(\begin{array}{c}
n+\alpha \\
n-2
\end{array}\right) \\
&+\quad \frac{M N}{(\alpha+1)(\alpha+2)}\left(\begin{array}{c}
n+\alpha \\
n-1
\end{array}\right)\left(\begin{array}{c}
n+\alpha+1 \\
n-2
\end{array}\right) .
\end{aligned}
$$

The coefficients $a_{0}(n, \alpha), \beta_{0}(n, \alpha)$ and $\gamma_{0}(n, \alpha)$ are respectively

$$
\begin{gathered}
a_{0}(n, \alpha)=\sum_{k=0}^{n}\left(\begin{array}{c}
k+\alpha \\
k-1
\end{array}\right)=\left(\begin{array}{c}
n+\alpha+1 \\
n-1
\end{array}\right), n=0,1,2, \ldots, \\
\beta_{0}(n, \alpha)=\sum_{k=1}^{n} \frac{k(\alpha+2)-(\alpha+1)}{(\alpha+1)(\alpha+3)}\left(\begin{array}{c}
k+\alpha \\
k-2
\end{array}\right)=\frac{n(\alpha+2)-\alpha}{(\alpha+1)(\alpha+4)}\left(\begin{array}{c}
n+\alpha+1 \\
n-2
\end{array}\right), \\
n=1,2,3, \ldots,
\end{gathered}
$$

and

$$
\gamma_{0}(n, \alpha)=\frac{1}{(\alpha+1)(\alpha+2)} \sum_{k=1}^{n}\left(\begin{array}{c}
k+\alpha \\
k-1
\end{array}\right)\left(\begin{array}{c}
k+\alpha+1 \\
k-2
\end{array}\right), n=1,2,3, \ldots .
$$

Note that the equations (45) and (46) can be written in the form

$$
(-1)^{k} \sum_{i=0}^{\infty} a_{i}(x) D^{i+k} L_{n}^{(\alpha)}(x)=\left(\begin{array}{c}
n+\alpha \\
n-k
\end{array}\right) D^{2} L_{n+1}^{(\alpha)}(x), k=0,1 .
$$


However it is even more remarkable that the equations (59), (60) and (61) can be written in the form

$$
\begin{aligned}
(-1)^{k+1} \sum_{i=0}^{\infty} c_{i}(x) D^{i+k} L_{n}^{(\alpha)}(x)=\frac{n(\alpha+k)-(\alpha+1)}{(\alpha+1)(\alpha+k+1)}\left(\begin{array}{c}
n+\alpha \\
n-2
\end{array}\right)\left(\begin{array}{c}
n+\alpha \\
n-k
\end{array}\right) D^{2} L_{n+1}^{(\alpha)}(x) \\
+\frac{2}{\alpha+1}\left(\begin{array}{c}
n+\alpha \\
n-2
\end{array}\right)\left(\begin{array}{c}
n+\alpha \\
n-k
\end{array}\right) D^{3} L_{n+1}^{(\alpha)}(x) \\
+\frac{1}{\alpha+1}\left(\begin{array}{c}
n+\alpha \\
n-k-1
\end{array}\right) \sum_{i=0}^{\infty} a_{i}(x) D^{i+2} L_{n}^{(\alpha)}(x) \\
+\left(\begin{array}{c}
n+\alpha \\
n-k
\end{array}\right) \sum_{i=0}^{\infty} b_{i}(x) D^{i+1} L_{n}^{(\alpha)}(x), k=0,1,2 .
\end{aligned}
$$

Also, we remark that the inversion formula of Lemma 5 can also be applied to (47) instead of (48) to obtain, by using (37) and (38),

$$
\begin{array}{r}
a_{i}(x)=(-1)^{i}\left[(\alpha+1) F_{i-1}(\alpha+2, \alpha+2 ; 2 ; x)-\frac{1}{\alpha+2} G_{i}(\alpha+2, \alpha ; 0 ; x)\right] \\
i=1,2,3, \ldots,
\end{array}
$$

which can be shown to be equal to (6) by straightforward but tedious computation.

Finally we remark that it can be shown that for $\alpha>-1$ and $\ell \in\{0,1,2, \ldots\}$

$$
\begin{aligned}
& \sum_{i=1}^{\infty} a_{i}(x) D^{i+\ell} L_{n}^{(\alpha)}(x) \\
& =-\left(\begin{array}{c}
n+\alpha \\
n
\end{array}\right) \sum_{k=0}^{\infty} \frac{(-n)_{k+\ell+1}(\alpha+3)_{k}}{(2)_{k}(\alpha+1)_{k+\ell+1} k !} \\
& \quad \times{ }_{3} F_{2}\left(\begin{array}{c}
-n+k+\ell+1,-\alpha-2, \alpha+k+3 \\
k+2, \alpha+k+\ell+2
\end{array}\right) x^{k+1},
\end{aligned}
$$

for $n=0,1,2, \ldots$

\section{REFERENCES}

1. H. Bavinck : A direct approach to Koekoek's differential equation for generalized Laguerre polynomials. Acta Mathematica Hungarica 66, 1995, 247-253. MR 96a:33009

2. H. Bavinck : A difference operator of infinite order with Sobolev-type Charlier polynomials as eigenfunctions. Indagationes Mathematicae, (N.S.) 7 (3), 1996, 281-291.

3. H. Bavinck \& H. van Haeringen : Difference equations for generalized Meixner polynomials. Journal of Mathematical Analysis and Applications 184, 1994, 453-463. MR 95h:33004

4. H. Bavinck \& R. Koekoek : On a difference equation for generalizations of Charlier polynomials. Journal of Approximation Theory 81, 1995, 195-206. MR 96h:33002

5. W.N. Everitt, L.L. Littlejohn \& R. Wellman : The symmetric form of the Koekoeks' Laguerre type differential equation. Journal of Computational and Applied Mathematics 57, 1995, 115-121. MR 96j:34148

6. I.H. Jung, K.H. Kwon, D.W. Lee \& L.L. Littlejohn : Sobolev orthogonal polynomials and spectral differential equations. Transactions of the American Mathematical Society $\mathbf{3 4 7}$, 1995, 3629-3643. CMP 95:14

7. I.H. Jung, K.H. Kwon, D.W. Lee \& L.L. Littlejohn : Differential equations and Sobolev orthogonality. Journal of Computational and Applied Mathematics 65, 1995, 173-180. MR 97a:33019

8. J. Koekoek \& R. Koekoek : On a differential equation for Koornwinder's generalized Laguerre polynomials. Proceedings of the American Mathematical Society 112, 1991, 1045-1054. MR 91j:33008 
9. R. Koekoek : Generalizations of the classical Laguerre polynomials and some q-analogues. Delft University of Technology, Thesis, 1990.

10. R. Koekoek: The search for differential equations for orthogonal polynomials by using computers. Delft University of Technology, report no. 91-55, 1991.

11. R. Koekoek: The search for differential equations for certain sets of orthogonal polynomials. Journal of Computational and Applied Mathematics 49, 1993, 111-119. MR 95m:33008

12. R. Koekoek \& H.G. Meijer : A generalization of Laguerre polynomials. SIAM Journal on Mathematical Analysis 24, 1993, 768-782. MR 94b:33007

13. T.H. Koornwinder : Orthogonal polynomials with weight function $(1-x)^{\alpha}(1+x)^{\beta}$ $+M \delta(x+1)+N \delta(x-1)$. Canadian Mathematical Bulletin 27(2), 1984, 205-214. MR 85i:33011

14. A.M. Krall : Orthogonal polynomials satisfying fourth order differential equations. Proceedings of the Royal Society of Edinburgh Sect. A 87, 1981, 271-288. MR 82d:33021

15. H.L. Krall : Certain differential equations for Tchebycheff polynomials. Duke Mathematical Journal 4, 1938, 705-718.

16. H.L. Krall : On orthogonal polynomials satisfying a certain fourth order differential equation. The Pennsylvania State College Studies, No. 6, 1940. MR 2:98a

17. H.L. Krall \& I.M. Sheffer : Differential equations of infinite order for orthogonal polynomials. Annali di Matematica Pura ed Applicata (4) 74, 1966, 135-172. MR 34:6260

18. Y.L. Luke: The Special Functions and Their Approximations II. Academic Press, New York, 1969. MR 40:2909

19. C.S. Meijer : Expansion theorems for the $G$-function I. Indagationes Mathematicae 14, 1952, 369-379. MR 14:469e

Delft University of Technology, Faculty of Technical Mathematics and Informatics, P.O. Box 5031, 2600 GA Delft, The Netherlands

E-mail address: r.koekoek@twi.tudelft.nl

E-mail address: h.bavinck@twi.tudelft.nl 